\title{
HOLE-DRILLING RESIDUAL STRESS MEASUREMENT IN AN INTERMEDIATE THICKNESS SPECIMEN \\ by
}

Colin Abraham

B.Sc., University of Santa Clara, 2009

A THESIS SUBMITTED IN PARTIAL FULFILLMENT OF THE REQUIREMENTS FOR THE DEGREE OF

MASTER OF APPLIED SCIENCE

in

THE FACULTY OF GRADUATE STUDIES

(Mechanical Engineering)

THE UNIVERSITY OF BRITISH COLUMBIA

(Vancouver)

September 2011

(C) Colin Abraham, 2011 


\section{Abstract}

The hole-drilling method for measuring residual stresses is generally applied to two extreme cases. In the "thick" case, the specimen depth is very large compared with the hole depth. The far boundary is then sufficiently distant that its effect can be neglected. In the "thin" case, the specimen has the form of a thin plate through which the hole penetrates fully. The thin plate creates plane-stress conditions and it is usually assumed that the associated residual stresses are uniform through the plate thickness. This research focuses on the intermediate case where the specimen has a thickness modestly greater than the hole depth. The far boundary is then near enough to give significant influence, and the through-thickness residual stresses are non-uniform. The finite specimen thickness and non-uniform residual stresses create significant bending deformations of the specimen during hole-drilling that are not present in either extreme case. This bending effect creates out-of-plane deformations that can provide an opportunity for a novel measurement approach. The "intermediate" case is investigated using an analytical model and by finite element analysis. Experimental measurements are made using Electronic Speckle Pattern Interferometry (ESPI), and a comparison is made of the theoretical and experimental results. 


\section{Table of Contents}

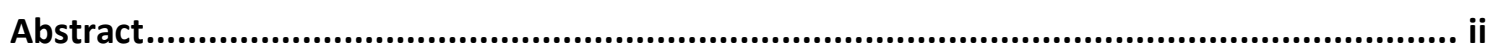

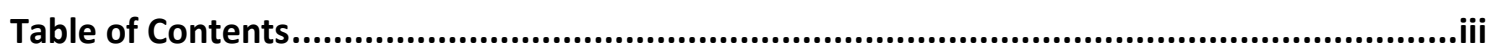

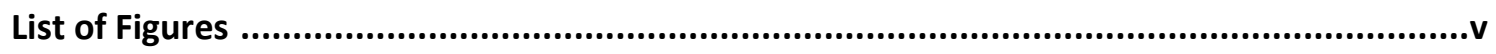

List of Symbols..................................................................................................................vii

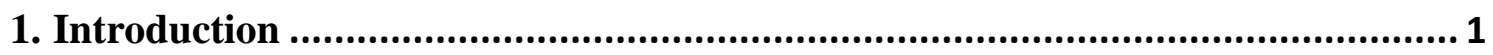

1.1. Residual Stresses ..................................................................................... 1

1.2. Current Measurement Techniques..................................................................... 1

1.2.1. Non-Destructive Tests ......................................................................... 2

1.2.2. Destructive Tests and Hole Drilling ........................................................ 2

1.2.3. Measurement Methods............................................................................. 3

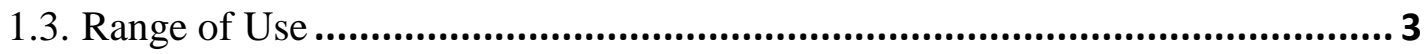

1.4. Introduction of Intermediate Case ................................................................. 4

1.5. Proposed Method and Objectives ........................................................................ 4

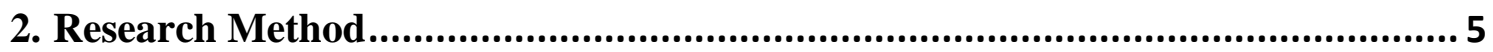

2.1. Finite Element Analysis (FEA) …………………......................................... 5

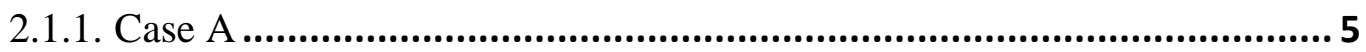

2.1.1.1. Axis-Symmetry ............................................................................6 6

2.1.1.2. Loads and Constraints ..................................................................... 6

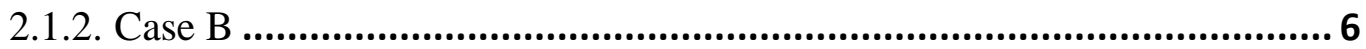

2.1.3. Out of Plane Bending.................................................................................. 7

2.2. Analytical Model .......................................................................................... 8 
2.2.1. Ring-Disk Approximation........................................................................ 8

2.2.2. Load Distribution .................................................................................... 9

2.2.3. Equations for Case A ............................................................................. 11

2.2.4. Equations for Case B .......................................................................... 19

2.2.5. Stress Profiling ...................................................................................... 28

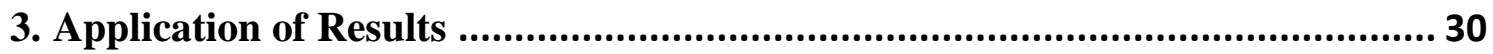

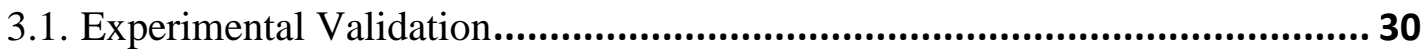

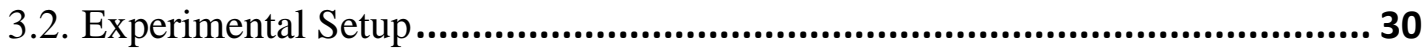

3.3. Electronic Speckle Pattern Interferometry …................................................. 34

3.4. Experimental Results ................................................................................... 41

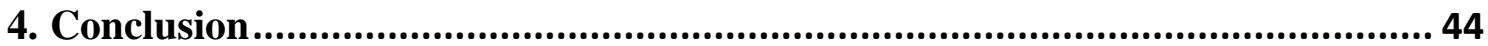

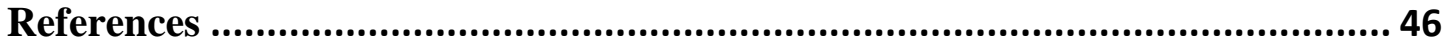




\section{List of Figures}

Figure 1: Residual stresses in a quenched metal bar ..............................................

Figure 2: FEA loads applied at inner edge of hole and constraints..............................6

Figure 3: Finite element analysis showing out of plane bending ................................

Figure 4: Calibration constant ā for a range of material thickness............................8

Figure 5: Deformed shape illustrating the associated bending deformation...................

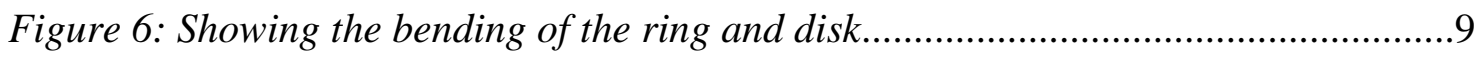

Figure 7: Defining the loads on the inner ring and outer disk ...................................

Figure 8: Defining the displacements of the system...............................................10

Figure 9: Demonstrating the contributions to horizontal surface displacement..............14

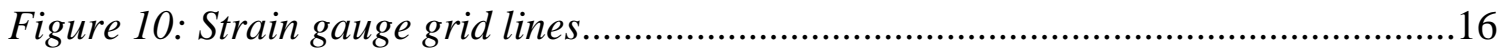

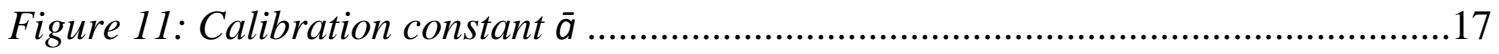

Figure 12: Showing weighting for interpolation function ...........................................18

Figure 13: Comparing mathematical solution and finite element solution ....................18

Figure 14: Out-of-plane relative displacements......................................................19

Figure 15: Calibration constant $万$ as relative part thickness increases.........................23

Figure 16: Displacements through part thickness radius for case $B$...........................24

Figure 17: Displacements through part thickness for case B (adjusted) .......................25

Figure 18: Calibration constant $\mathrm{5}$ as thickness increases (adjusted) ...........................26

Figure 19: Calibration constant $Ђ$ as thickness increases (interpolated) .....................27

Figure 20: Relative out-of-plane displacements for Ђ as thickness increases. ..............27

Figure21: Average stress measurements and stress profiling measurements ..............28

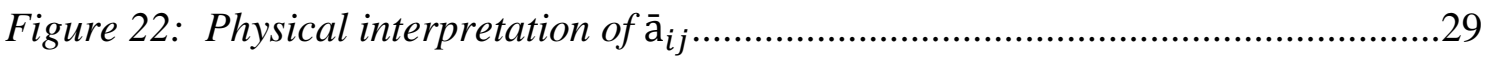

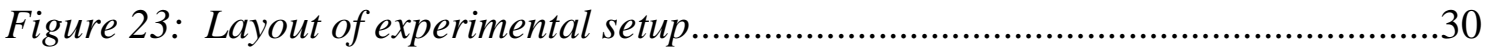


Figure 24: Specimen holder.

Figure 25: Bending of specimen showing linearly varying stresses..............................

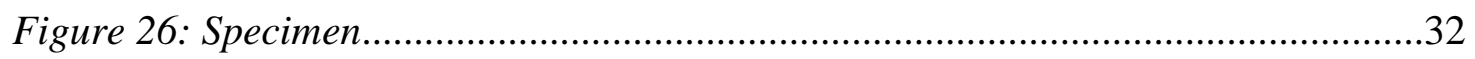

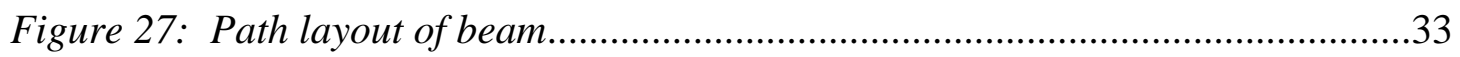

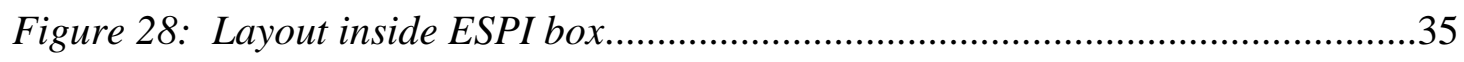

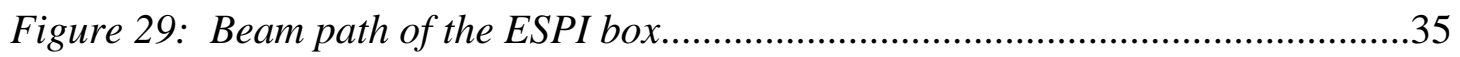

Figure 30: Ray diagram for ESPI speckles.......................................................36

Figure 31: Set of four reference images for ESPI measurement................................37

Figure 32: Set of four measurement images for ESPI measurement..........................38

Figure 33: Using phase stepping and intensity to compute phase change. .................39

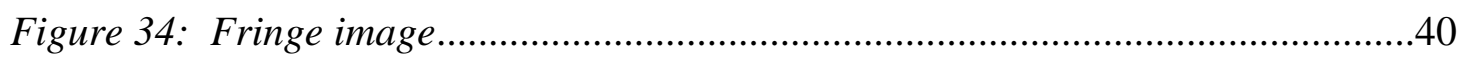

Figure 35: Fringe image with interpolation of bad pixels and outliers.....................41

Figure 36: Experimental results for stress in the $X$ direction..................................42 


\section{List of Symbols}

a Hole radius

$\overline{\mathrm{a}} \quad$ Calibration constant for case A

$\overline{\mathrm{a}}_{\mathrm{ij}} \quad$ Strain relaxation due to a unit stress within increment $j$ of a hole $i$ increments deep

万 Calibration constant for case B

C Net force acting on outer ring

D Bending elastic stiffness term from text

$\Delta \quad$ Horizontal motion at center of outer ring at ring-disk boundary

$\delta \quad$ Horizontal motion at center of inner disk at ring-disk boundary

E Young's modulus

$\varepsilon \quad$ Strain

$\varepsilon_{\mathrm{i}} \quad$ Measured strain relaxation after the $i$ th hole depth increment

F Net radial force

h Hole depth

H Stress depth

I Combined intensity

$\mathrm{I}_{\mathrm{a}} \quad$ Reference beam intensity

$\mathrm{I}_{\mathrm{b}} \quad$ Object beam intensity

$\phi \quad$ Phase for object beam

$\phi_{\mathrm{R}} \quad$ Phase for reference beam

M Moment acting on inner disk

$n \quad$ Number of hole depth increments

P Pressure equivalent of relieved residual stresses

Q Net moment acting on outer ring

r Radial coordinate

$r_{\text {curve }}$ Bend radius for specimen 
S Force equivalent of relieved residual stresses

$\sigma_{\mathrm{x}} \quad$ Normal Stress in $\mathrm{X}$ direction

$\sigma_{\mathrm{y}} \quad$ Normal Stress in Y direction

$\sigma_{\mathrm{r}} \quad$ Normal Stress in radial direction

$\sigma_{\mathrm{t}} \quad$ Normal Stress in tangential direction

$\sigma_{\mathrm{A}} \quad$ Equi-biaxial stress field, $\sigma_{\mathrm{A}}=\sigma_{\mathrm{x}}=\sigma_{\mathrm{y}}$

$\sigma_{\mathrm{B}} \quad$ Shear stress field, $\sigma_{\mathrm{B}}=\sigma_{\mathrm{x}}=-\sigma_{\mathrm{y}}$

$\sigma_{\mathrm{j}} \quad$ Equivalent uniform stress within the $j$ th hole depth increment

$\varphi_{\mathrm{r}} \quad$ Angular deflection in radial direction

$\varphi_{\theta} \quad$ Angular deflection in circumferential direction

$\theta \quad$ Angular coordinate

$\tau_{\mathrm{xy}} \quad$ Shear stress

$v \quad$ Poisson's ratio

W Part thickness

Y Distance from bending centerline 


\section{Chapter 1 Introduction}

\subsection{Residual Stress}

Residual stresses are self-equilibrating stresses that exist within a material without an externally applied load. They are often formed in the manufacturing process, for example by milling, forging, welding, and rapid temperature changes [1]. Consider the example of a metal rod at high temperature that is being quenched. As the outside of the rod cools rapidly it will contract causing a tensile stress in the outer edge of the rod (figure 1a). This stress may cause some localized yielding in the outer edge of the rod as the stresses attempt to equalize (figure 1b). Then as the interior of the rod starts to cool and shrink the outside of the rod becomes subject to a longitudinal compressive stress while the interior experiences a self-equilibrating tensile stress (figure 1c).

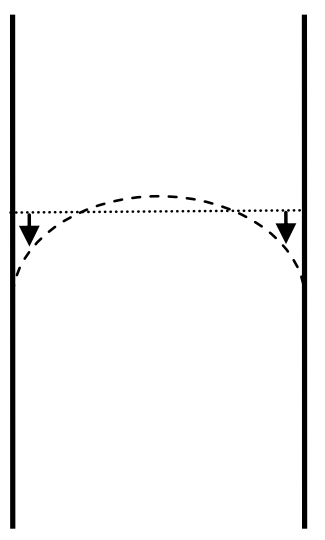

(a)

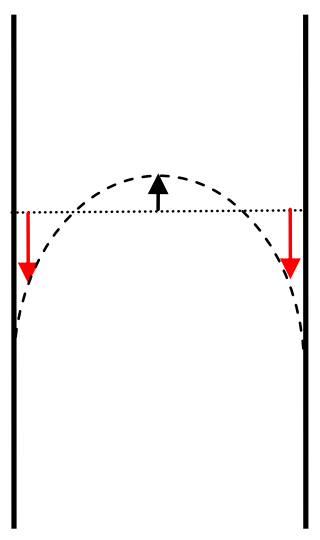

(b)

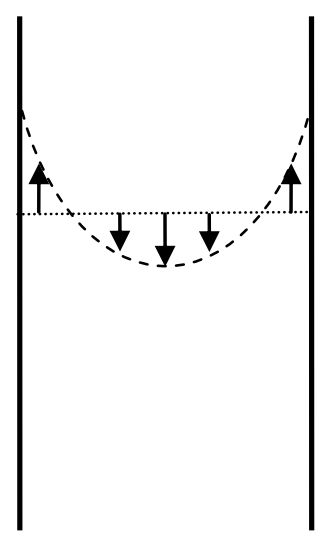

Compression

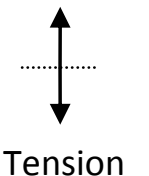

(c)

Figure 1: Showing the formation of residual stresses in a quenched metal bar.

High levels of residual stresses also create problems when working with material. When milling highly stressed material, material removal causes the rest of the part to deform as the remaining stresses equalize. This makes it very difficult to maintain accuracy when working with such parts, causing loss of time and material [2]. Some residual stresses can be beneficial and are deliberately introduced. Shot peening purposefully induces high levels of compressive residual stresses in the surface layer of a part in order to increase fatigue resistance. No matter the origin of the residual stresses it is imperative to be able to determine the magnitude and direction of these stresses accurately.

\subsection{Current Measurement Techniques}

Because they exist without any external load, residual stresses cannot be measured by conventional stress measurement techniques. There are several specialized 
methods for measuring residual stresses. These methods vary greatly in accuracy, ease of implementation and the depth into the part to which they can measure.

\subsubsection{Non-Destructive Tests}

As the name implies, non-destructive residual stress measurement techniques do not damage the target part during measurement. These are non-contact techniques that include X-ray diffraction, neutron diffraction and ultrasonic methods. These methods cause no damage to the work piece, but the results are often limited to those stresses very close to the surface of the part or else require major capital equipment.

Diffraction methods rely on the change in crystal structure of a material under a load. The workpiece is exposed to radiation close to the interplanar spacing of the crystal structure and the resulting scatter is imaged by a detector. Using the observed scatter the stresses can be calculated using Bragg's Law [3]. This method allows for both the magnitude and principal direction of the stresses to be measured with no damage to the part. This technique does have some drawbacks. Firstly the use of X-ray or neutron radiation makes certain safety precautions necessary and makes it a very equipment intensive technique. This method also suffers from very limited depth of measurement. When using X-rays, only those stresses in the surface of the part that can be penetrated by the radiation can be measured, for metals this is just a few microns. Neutron diffraction measurements can identify deep interior stresses, but require the use of a specialized nuclear reactor.

The ultrasonic method can also be used to measure residual stresses. This method relies on the fact that elastic wave propagation in a solid depends on the mechanical stress within the solid. An emitter generates ultrasonic waves that can then be detected and the stress calculated from the propagation characteristics [4]. This method is popular for measuring the strength of welds as no damage is done to the part and test equipment can be made fairly portable. However, the technique requires detailed calibration and the results have only modest accuracy.

\subsubsection{Destructive Tests and Hole Drilling}

There are several destructive methods to measure residual stresses. The most common residual stress measurement method is the hole drilling method. The hole drilling method involves drilling a small hole in the surface of a part and measuring the deformation of the surrounding surface and is detailed in the ASTM standard E-837 [5]. This method is known as a semi-destructive method because the damage caused by drilling a small hole often does not seriously affect the functionality of the part.

The hole drilling residual stress measurement method has been in use for many years. Mathar first proposed the idea in the 1930s and since then the methods and 
application have developed extensively [6]. There are several variations to this technique. Slitting, excising and ring coring are fundamentally the same idea as hole drilling. They all involve removing stressed material and measuring the local response of the material [7].

\subsubsection{Measurement Methods}

The surface deformations can be measured in several ways. Strain gauges are often used as the required equipment is relatively cheap and easy to set up and gives reliable results. There are special three and six element strain gauge rosettes for use with hole drilling. One drawback to using strain gauges is that they need to be glued to the surface of the part. When a great number of tests are being done their cost and installation time can become quite significant.

Several different optical methods can be used to measure the deformations of a drilled surface. For example, Electronic Speckle Pattern Interferometry (ESPI) can be used to provide full-field measurements of surface deformations. The sensitivity of this method depends on the wavelength of light being used, typically some hundreds of nanometers. This method involves shining coherent laser light on a surface and imaging it with a CCD camera. A hole is then drilled and the surface re-imaged. The motion of the surface can then be computed by resulting phase change [8]. This method has several advantages: it is a full-field, non-contact method, and it can detect both in plane and out of plane motion of the surface.

\subsection{Range of Use}

The hole drilling method has been applied to two distinct cases. The first case is the 'thin' case where the hole penetrates the entire thickness of the part. This is used for thin parts, such as sheet metal or thin plates. The analytical solution to this case is given by the classical Kirsch solution and can be readily calculated. With current procedures, stress profiling is not possible in this case.

The second case is the 'thick' case in which the thickness of the part is large compared with the hole diameter. A minimum thickness of 1.2 times the diameter of the gauge circle is recommended by the ASTM standard [5]. In this case the part can be treated as being infinitely thick so all the deformations that occur from the drilling of the hole happen near the part and are not influenced by the part thickness. The calibration constants for this case have been found experimentally and with finite element analysis 
and are listed in the ASTM standard. Stress profiling is possible for this case because there is no dependency on the depth of the hole in relation to the total part thickness.

\subsection{Introduction of Intermediate Case}

Many parts in the real world do not fall into either the thin or the thick categories. The far boundary is often likely to be close enough to play a significant role in the behaviour of the response. This demonstrates the need for a hole drilling solution to the intermediate thickness work pieces. Moreover, it is found that the behaviour of the intermediate case has elements that are not seen in either the thick or the thin case, which would lead to serious error if the cases are simply interpolated between the extreme cases, as is the implicit suggestion in ASTM E837.

In the intermediate case there exists significant bending deformation. This behaviour is not seen in either of the two previous cases. In the thin case this is not present because the part is symmetric. In the thick case, the substantial thickness of the part inhibits any significant bending. This bending dominates the response for cases just thicker than the hole depth and makes a simple interpolation scheme infeasible. This bending behaviour also provides critical insight needed to develop a mathematical model for the intermediate part.

\subsection{Proposed Method and Objectives}

The objective of this research is to enable application of the hole drilling method to specimens of any relative thickness, not just the two extreme cases. The approach taken uses finite element analysis to conduct computer simulation of the behaviour of the intermediate case. Numerical models of the behaviour are developed from these. This method conveys the needed calibration constants through analytical equations which present the information much more compactly than if tables were made for each relative thickness. The proposed method should also allow stress profiling on thin plates, which was not previously possible. 


\section{Chapter 2 Research Method}

This research uses a three-stage approach. First the behaviour of the intermediate case is analysed using finite-element simulation. This allows the case to be explored and to physically understand what is happening. The second stage is to derive analytical equations that will allow the results to be applied easily. The objective is to avoid the need for an extensive set of empirical calibration tables. As an alternative to this, analytical equations are derived to reduce the use of tables needed to a more manageable amount. The final stage is to perform physical experiments to validate the new method. This will ensure that the finite element analysis and mathematical approximations are valid for practical applications.

\subsection{Finite Element Analysis (FEA)}

Finite element analysis is used in this research for its ability to produce accurate data for many different cases accurately and to develop a deep intuitive understanding of the behaviour of the intermediate thickness specimen. This intuitive understanding was critical to the success of the research because it helped identify the controlling behaviour described by the mathematical model.

\subsubsection{Case A}

Two calibration constants are needed for the hole drilling method. The two constants are $\bar{a}$ and $\bar{b}$, indicated by the red arrows. These constants relate the measured strains to the residual stresses as seen in equation 1 . The need for these constants arises from the fact that the strains, $\varepsilon$, are not measured at the location of the stresses, $\sigma_{\mathrm{y}}, \sigma_{\mathrm{x}}$ and $\tau_{\mathrm{xy}}$. E is defined as Young's modulus, $v$ is Poisson's ratio, and $\theta$ is the angle from the principle stress direction.

$$
\varepsilon=\frac{\grave{a}}{E}\left((1+v) \frac{\left(\sigma_{\mathrm{x}}+\sigma_{y}\right)}{2}\right)+\frac{\downarrow}{E}\left(\frac{\left(\sigma_{\mathrm{x}}-\sigma_{y}\right)}{2} \cos 2 \theta+\tau_{x y} \sin 2 \theta\right)
$$

The first term of equation 1 relates the axisymmetric stresses to the measured strain. This is termed the " $\mathrm{A}$ " case. The second term of the equation similarly relates the 45-degree shear stress and the axial shear stress. This is termed the " $\mathrm{B}$ " case and will be discussed later. 


\subsubsection{Axis-Symmetry}

The A case exhibits rotational symmetry about the center of the hole. This allows the analysis to be reduced to a 2-D problem for which axisymmetric finite elements can be used. By reducing the problem from a 3-D to a 2-D problem, the model is simplified and the computation time substantially reduced.

\subsubsection{Loads and Constraints}

To simulate the residual stresses in a workpiece, a load is applied at the inner edge of the hole, as indicated by the arrows in figure 2. By superposition, this is shown to be an appropriate method to model the stresses relieved by hole drilling [9]. The inner edge of the model must be constrained in the radial direction and the outer edge must be pinned in the normal direction to fully constrain the model. The model is 40 times the hole radius wide and varies in thickness from the hole depth to 20 times the hole depth. These outer boundaries are found to be sufficiently far as increasing it further has no impact.

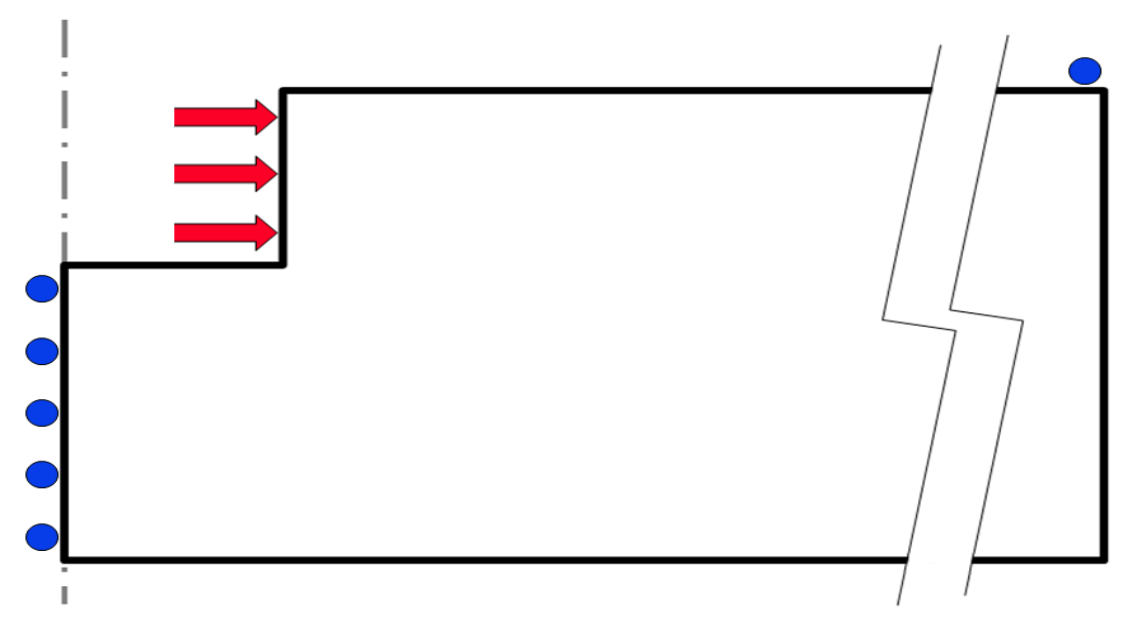

Figure 2: Diagram of FEA analysis showing loads applied at inner edge of hole and constraints.

\subsubsection{Case B}

The second term in equation 1 describes the effect of shear stresses. This is termed the "B" case and uses the calibration constant $\mathrm{b}$ to relate the measured strains to the stresses. This case can be examined the same way that case A is but with the addition of $\cos 2 \theta$ and $\sin 2 \theta$ terms. All deformations have the same trigonometric variations and 
so the model can still be reduced to a two dimensional problem. Specialized, "axiharmonic" finite elements are available to handle this case. The finite element analysis is performed similar to case A with the appropriate changes.

\subsubsection{Out of Plane Bending}

When the intermediate specimen is analysed, a behaviour not seen in either the thin case or the thick case is present. Out-of-plane bending occurs which is seen in neither of the previous cases, and shown in figure 3. This bending is caused by the loss of symmetry exhibited in the thin case and the lack of stiffness of the thick case.

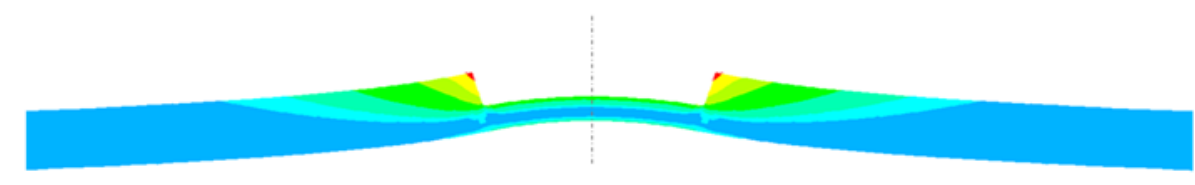

Figure 3: Finite element analysis showing out of plane bending for a workpiece just thicker than the thin case.

The out-of-plane bending affects the response of the A case as it transitions from thin, through to intermediate and then to thick. Specifically, the bending augments the displacements, which in turn increases the calibration constant ā. Figure 4 shows the variation of calibration constant $\bar{a}$ with specimen thickness for four different hole depths. The points at the left side of the graph correspond to the "thin" case. They are all equal because the stress distribution around the drilled hole is independent of plate thickness. The points at the right side of the graph correspond to the "thick" case. Both of these sets of points are separately tabulated in ASTM Standard Test Procedure E-837. Figure 4 shows that the "intermediate" behaviour is not a simple interpolation of the extreme cases. The prominent peaks on the left side of the graph are caused by the out-of-plane bending illustrated in Figure 3. 


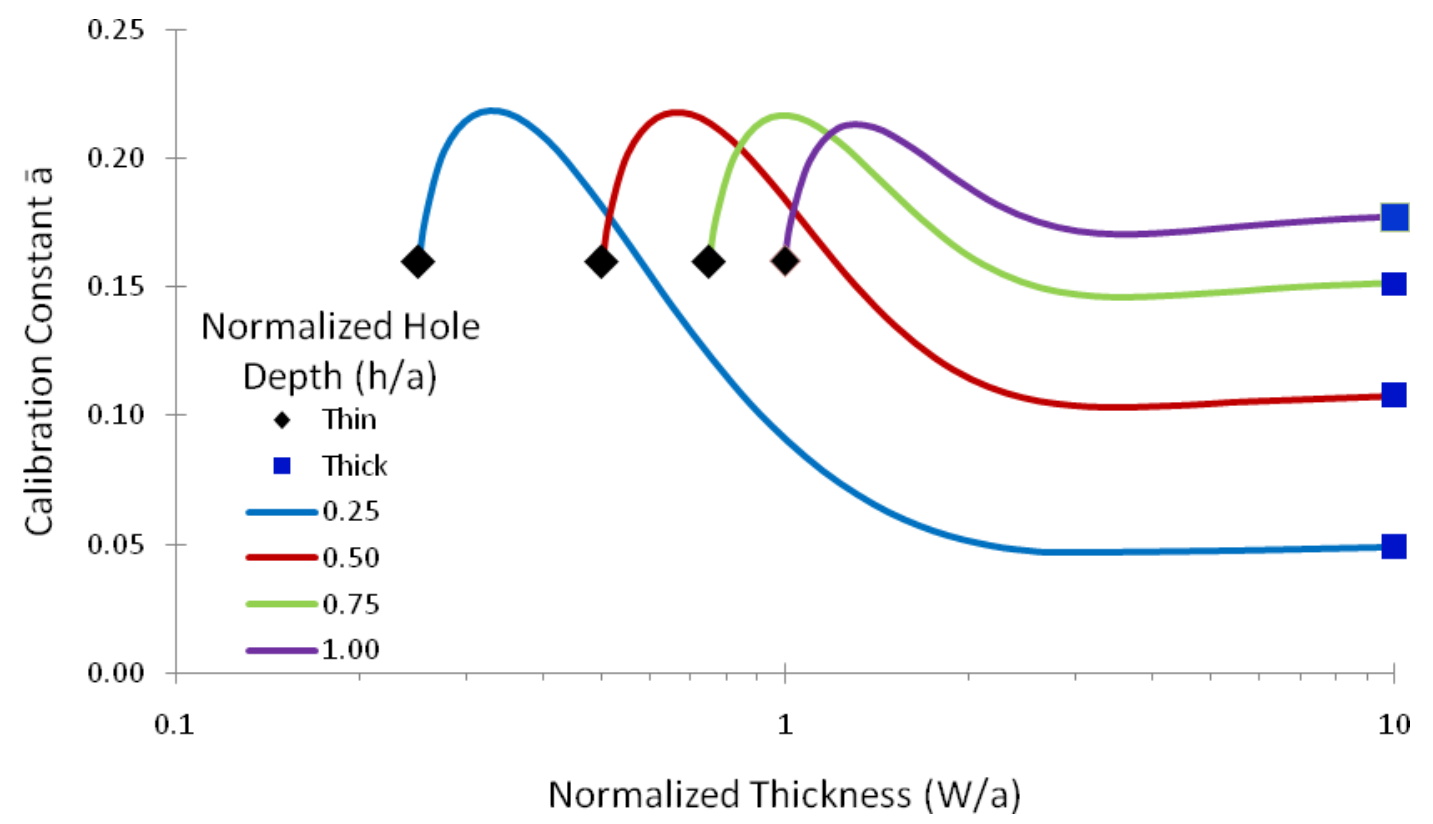

Figure 4: Calibration constant ā for a type " $A$ ' strain gange rosette (ASTM E837) for a range of material thickness. Previously known calibration constants are plotted at the two extreme ends and show good agreement with the finite element analysis.

\subsection{Analytical Model}

Currently the calibration constants are listed in the ASTM standard in sets of tables. This works for the current application because there is only a dependence on the hole diameter, depth and rosette type used. This means that the calibration constants can be shown effectively within a compact set of tables. However, in the intermediate case a fourth dependence is created, specifically the part thickness. This adds a new dimension that greatly multiplies the number of tables required, thus making this approach impractical. By deriving analytical equations that convey this information, it is possible to handle the intermediate case using the equation and existing tables only, and without requiring any further tables.

\subsubsection{Ring-Disk Approximation}

The behaviour of this model is complex so simplifying assumptions must be made in order to create a workable equation. Using the insight from the finite element analysis, to the plate and hole are modeled as an inner disk surrounded by an outer ring, as shown in Figures 5 and 6. 


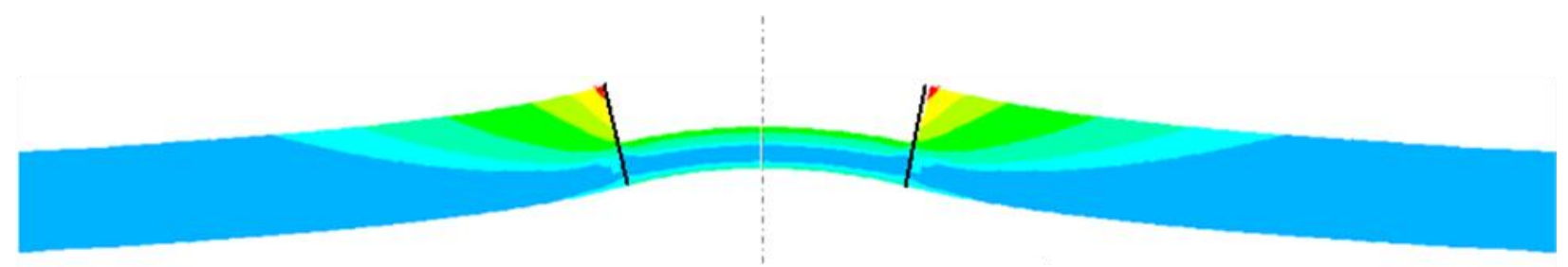

Figure 5: Deformed shape of a thin plate with drilled hole, illustrating the associated bending deformation.
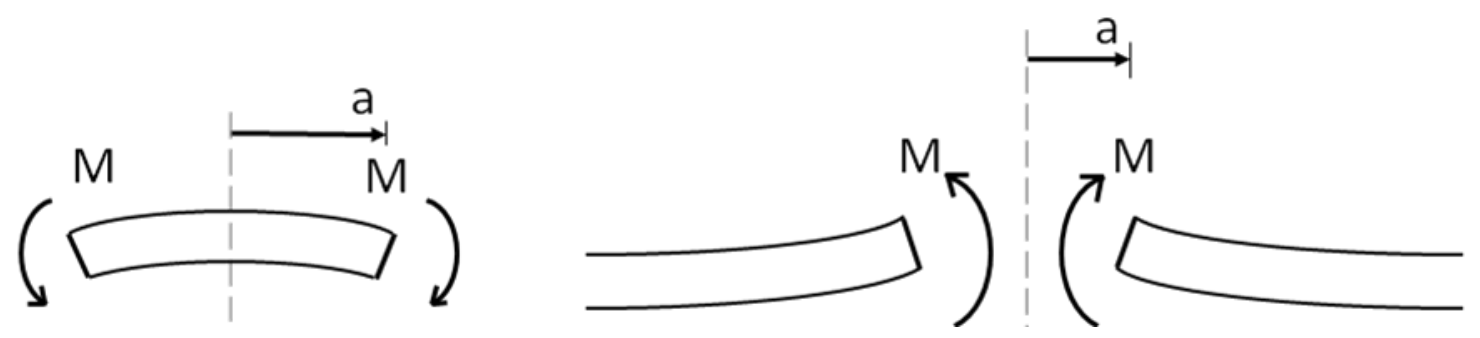

Figure 6: Showing the bending of the ring and disk.

\subsubsection{Load Distribution}

The reaction of the part is a combination of both the horizontal displacement and the bending. The load is shared by both the inner disk and the outer ring. Forces and moments are shown in figure 7. Equations are derived for the load balance of the part to enforce equilibrium.
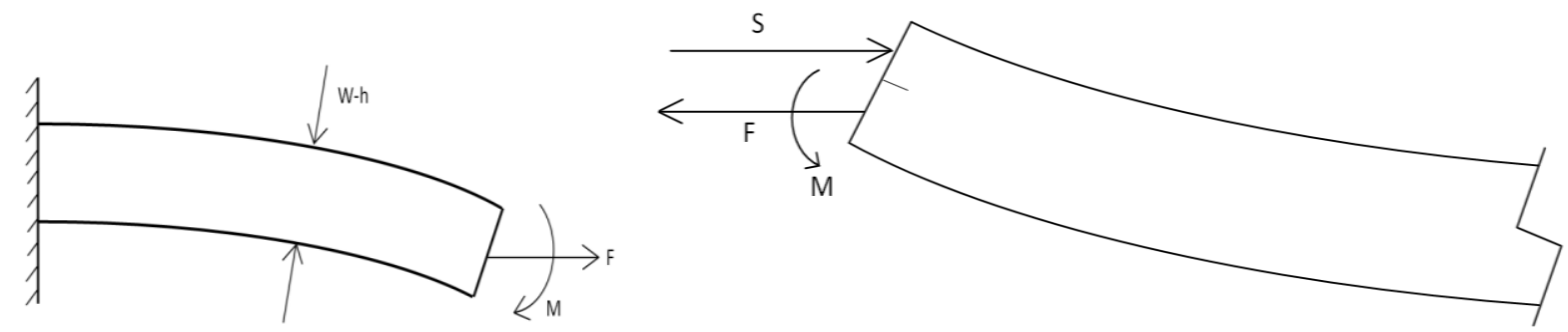

Figure 7: Defining the loads on the inner ring and outer disk. 
Equivalent radial force, $\mathrm{S}$, from the pressure, $P$, on the inner wall, where $h$ is the hole depth and $a$ is the hole radius.

$$
S=P h a
$$

Net radial force on inner disk

Net radial force on outer ring

$$
C=S-F
$$

Net moment acting on inner disk

$$
\text { M }
$$

Net moment acting on outer ring

$$
Q=S\left(\frac{W-H}{2}\right)+F\left(\frac{h}{2}\right)-M
$$
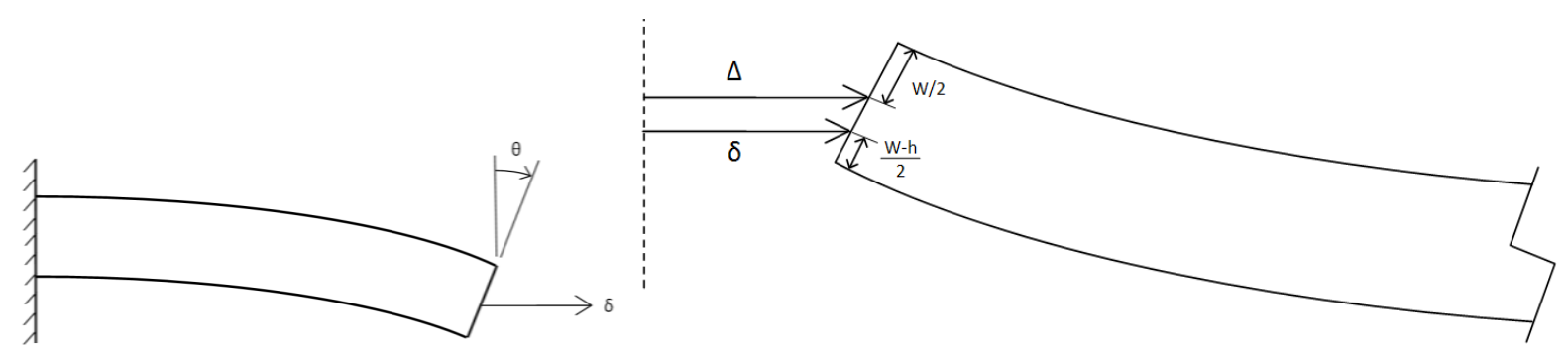

Figure 8: Defining the displacements of the system.

The horizontal motion at center of outer ring at the ring-disk boundary is defined as $\Delta$, and the horizontal motion at center of inner disk at the ring-disk boundary is defined as $\delta$, as shown in figure 8 . These can be related to each other by equation 5 .

$$
\Delta=\delta+\frac{h \theta}{2}
$$


Elastic stiffness for the inner disk and outer ring are defined as $\mathrm{k} 1$ and $\mathrm{k} 2$ respectively. Where small $\mathrm{k}$ represents the horizontal stiffness and big $\mathrm{K}$ represents the bending stiffness.

$$
\begin{array}{ll}
F=k_{1} \delta & M=K_{1} \theta \\
C=k_{2} \Delta & Q=K_{2} \theta
\end{array}
$$

Using equations 3,4 and 5, $\delta$ and $\theta$ are solved for

$$
\begin{aligned}
& \theta=S\left[\frac{2 k_{1} h+2\left(k_{1}+k_{2}\right)(W-H)}{k_{1} k_{2} h^{2}+4\left(k_{1}+k_{2}\right)\left(K_{1}+K_{2}\right)}\right] \\
& \delta=S\left[\frac{\mathbf{4}\left(K_{1}+K_{2}\right)-k_{2} h(W-H)}{k_{1} k_{2} h^{2}+4\left(k_{1}+k_{2}\right)\left(K_{1}+K_{2}\right)}\right]
\end{aligned}
$$

Given $Q=K_{\mathbf{2}} \theta$ the net moment acting on outer ring is:

$$
Q=S\left[\frac{2 k_{1} h K_{2}+2 K_{2}\left(k_{1}+k_{2}\right)(W-H)}{k_{1} k_{2} h^{2}+4\left(k_{1}+k_{2}\right)\left(K_{1}+K_{2}\right)}\right]
$$

The net horizontal force $\mathrm{C}$ is similarly:

$$
C=S\left[\frac{4 k_{2}\left(K_{1}+K_{2}\right)+k_{1} k_{2} h(W+h-H)}{4\left(k_{1}+k_{2}\right)\left(K_{1}+K_{2}\right)+k_{1} k_{2} h^{2}}\right]
$$

For case A the stiffness values are given below:

$$
\begin{array}{ll}
k_{1}=\frac{(W-h) E}{1-v} & k_{2}=\frac{W E}{1+v} \\
K_{1}=\frac{(W-h)^{3} E}{12(1-v)} & K_{2}=\frac{W^{3} E}{12(1+v)}
\end{array}
$$

\subsubsection{Equations for Case A}

Four separate equations are needed. Equations are required for the bending of the inner disk and outer ring, as well as for the radial displacement of the inner disk and outer 
ring. These equations can then be combined to evaluate the load distribution and finally the surface displacements.

For the A case the analytical equation for the outer ring's horizontal displacement is easily calculated. The equation comes from the horizontal displacement of a thick cylinder with a pressure applied at the inner edge.

The in-plane stresses in the radial and tangential directions are described by the Lamé equations:

$$
\begin{aligned}
& \sigma_{r}=C_{1}+\frac{C_{2}}{r^{2}} \\
& \sigma_{t}=C_{1}-\frac{C_{2}}{r^{2}}
\end{aligned}
$$

For the case with a pressure, $P$, applied at the inner edge, substitute the boundary conditions $\sigma_{r}=-P$ at $\mathrm{r}=\mathrm{a}$ and $\sigma_{r}=0$ at $\mathrm{r}=\infty$ gives

$$
\begin{gathered}
\sigma_{r}=-\frac{a^{2} P}{r^{2}} \\
\sigma_{t}=\frac{a^{2} P}{r^{2}}
\end{gathered}
$$

Applying Hooke's law:

$$
\varepsilon_{t}=\frac{\sigma_{t}}{E}-\frac{v \sigma_{r}}{E}
$$

and noting that for axi-symmetric geometry the radial displacement is

$$
U_{r}=r \varepsilon_{t}
$$

Then the radial displacement for the outer ring is

$$
U_{r}=\frac{P a^{2}}{E r}(1+v)
$$


The equations for the bending of the outer ring can be found in [10]. The case described in the test is a ring with an inner radius of $a$ and an outer radius of $b$. This ring is then loaded by a moment $r$ at the inner boundary.

$$
\varphi=M\left[\frac{\left(-b^{2}(1+v)-(1-v) a^{2}\right)\left(a^{2}(1+v)+r^{2}(1-v)\right)+\left(r^{2}-a^{2}\right)\left(1-v^{2}\right)\left(b^{2}-a^{2}\right)}{2 D r\left(1-v^{2}\right)\left(b^{2}-a^{2}\right)}\right]
$$

The case of interest for this analysis is the infinite case where b goes to infinity.

$$
\begin{gathered}
\varphi=M\left[\frac{-(1+v)\left(a^{2}(1+v)+r^{2}(1-v)\right)+\left(r^{2}-a^{2}\right)\left(1-v^{2}\right)}{2 D r\left(1-v^{2}\right)}\right] \\
\varphi=M\left[\frac{-a^{2}(1+v)^{2}-r^{2}\left(1-v^{2}\right)+\left(r^{2}-a^{2}\right)\left(1-v^{2}\right)}{2 D r\left(1-v^{2}\right)}\right] \\
\varphi=\frac{-M}{r} \frac{a^{2}}{D(1-v)}
\end{gathered}
$$

Where D is the elastic stiffness

$$
D=\frac{E W^{3}}{12\left(1-v^{2}\right)}
$$

This gives the angular deflection as a function of $r$

$$
\varphi=\frac{-M}{r} \frac{a^{2} 12(1+v)}{E W^{3}}
$$

Integrating this gives the vertical displacement 


$$
\begin{gathered}
Y=\int \varphi d r+C_{1} \\
Y=\int \frac{-M}{r} \frac{a^{2} 12(1+v)}{E W^{3}} d r+C_{1} \\
Y=\ln (r) \frac{-M a^{2} 12(1+v)}{E W^{3}}+C_{1}
\end{gathered}
$$

Setting the vertical displacement at the hole edge equal to 0

$$
\begin{gathered}
Y(a)=0 \\
0=\ln (a) \frac{-M a^{2} 12(1+v)}{E W^{3}}+C_{1} \\
C_{1}=\ln (a) \frac{-M a^{2} 12(1+v)}{E W^{3}} \\
Y=\ln (r) \frac{-M a^{2} 12(1+v)}{E W^{3}}-\ln (a) \frac{-M a^{2} 12(1+v)}{E W^{3}}
\end{gathered}
$$

Combining terms gives the vertical displacement as a function of radius

$$
\mathrm{Y}=\ln \left(\frac{r}{a}\right) \frac{-M a^{2} 12(1+v)}{E W^{3}}
$$

The in-plane displacements come from two sources: the horizontal displacement of the outer ring and from the out-of-plane bending of the ring. These contributions are illustrated in figure 9.
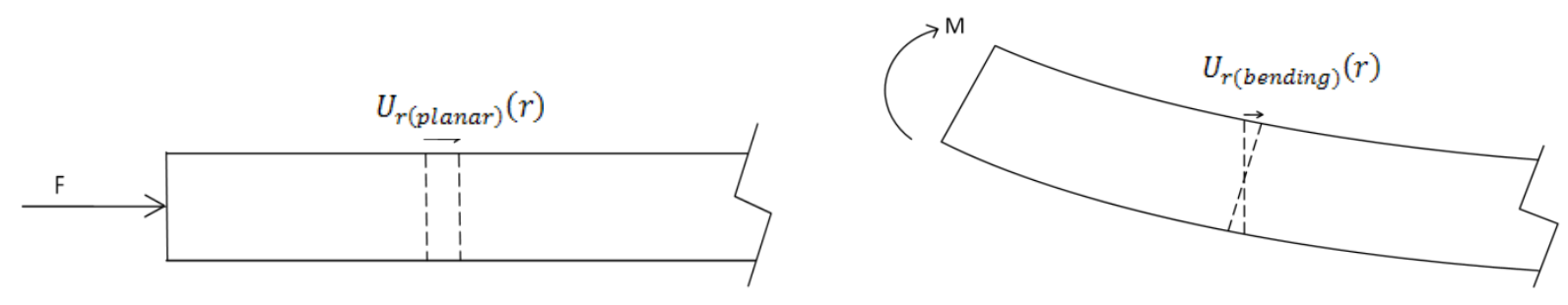

Figure 9: Demonstrating the contributions to horizontal surface displacement.

The net force and net moment acting on the outer ring are given by $\mathrm{Q}$, the net moment (eq 10) and C, the net force (eq 11). The displacement from the horizontal displacement of the outer ring is given by: 


$$
U_{r(\text { planar })}(r)=\frac{C a}{H E r}(1+v)
$$

The portion of the radial displacement contributed by the bending of the ring is given by the sin of the angular deflection times the thickness of the part.

$$
U_{r(\text { bending })}(r)=\frac{W}{2} \sin 2 \varphi
$$

This term is then simplified by taking the Taylor expansion of the sin term.

$$
U_{r(\text { bending })}(r)=\frac{W}{2}\left(\varphi-\frac{\varphi^{3}}{3 !}+\frac{\varphi^{5}}{5 !} \ldots\right)
$$

Dropping all but the first term gives

$$
U_{r(\text { bending })}(r)=\frac{W}{2} \varphi
$$

Theta at any radial location is given by the equation

$$
\varphi=\frac{-M}{r} \frac{a^{2} 12(1+v)}{E W^{3}}
$$

Substituting Q (net moment) in for M and combining with equation 36

$$
U_{r(\text { bending })}(r)=\frac{W}{2}\left[\frac{-Q}{r} \frac{a^{2} 12(1+v)}{E W^{3}}\right]
$$

Combining the two contributions gives the total radial displacement as a function of $r$ using the load distribution from equations 10 and 11

$$
U_{r}(r)=\frac{C a}{H E r}(1+v)+\frac{W}{2}\left[\frac{-Q}{r} \frac{a^{2} 12(1+v)}{E W^{3}}\right]
$$

This equation can then be used to calculate the known value $\bar{a}$ using the method described in [11]. Equation 40 calculates the strain gauge response from displacement data. 


$$
\varepsilon^{*}=\frac{\sum_{j=1}^{n} \frac{U\left(X_{2(j)}, Y_{(j)}\right)-U\left(X_{1(j)}, Y_{(j)}\right)}{W_{(j)}}}{\sum_{j=1}^{n} \frac{\left(X_{2(j)}-X_{1(j)}\right)}{W_{(j)}}}
$$

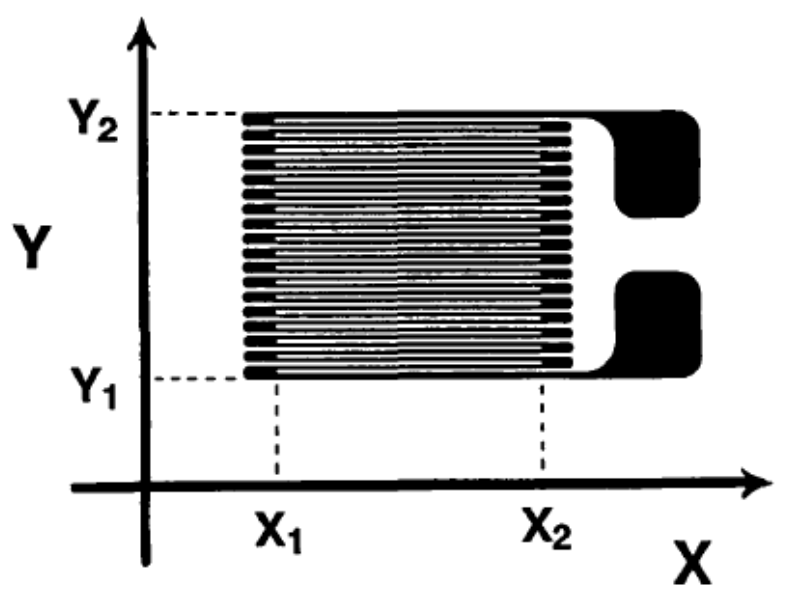

Figure 10: Strain gauge grid lines. The strain is calculated by summing the displacements at each end point as described in equation 40.

Where $Y_{(j)}$ is the value of grid line $n$ and $X_{1(j)}$ and $X_{2(j)}$ are the values of the end points as can be seen in figure 10. The displacements are then calculated at each end point and summed to compute $\varepsilon^{*}$ then from there $\bar{a}$ can be calculated using equation 41 . Where $\sigma_{\mathrm{A}}$ is the equi-biaxial stress field, $\sigma_{\mathrm{A}}=\sigma_{\mathrm{x}}=\sigma_{\mathrm{y}}$.

$$
\overline{\mathrm{a}}=\frac{\varepsilon^{*}}{2 \sigma_{A}}
$$




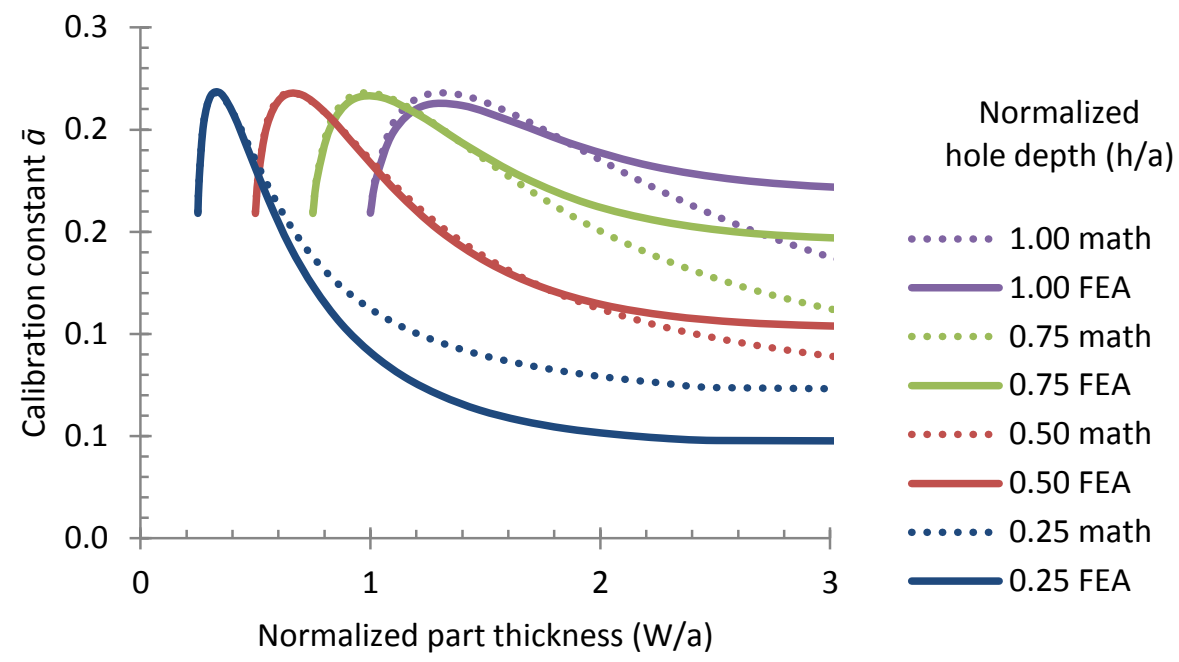

Figure 11: Comparison of calibration constant à for mathematical solution and finite element solution.

The mathematical solution agrees well for relatively thin parts, but does not agree with the finite element analysis as the part thickness gets large in relation to hole depth. This is illustrated in figure 11. The cause of this discrepancy comes from the simplifying assumptions. The mathematical model treats the workpiece as a thin plate. As the thickness becomes great the deformations all occur locally to the hole edge. This is not problematic, because the response for the thick case is already well known. A non-linear interpolation function is used to bridge thin and thick cases.

$$
\begin{aligned}
& A\left(\frac{h}{W}\right)=f\left(\frac{h}{W}\right) A_{\text {analytic }}+\left(1-f\left(\frac{h}{W}\right)\right) A_{A S T M} \\
& \text { Where } \quad f\left(\frac{h}{W}\right)=\exp \left(-0.2 \frac{h}{a}\left(\frac{W}{h}-1\right)^{2}\right)
\end{aligned}
$$

The interpolation function was chosen because it spans the range 1 to 0 as $\mathrm{W} / \mathrm{h}$ varies from 1 to infinity. Near $\mathrm{W} / \mathrm{h}=1$, the function has unit value with zero gradient to allow the mathematical solution to apply over a significant range of small W/h. For large $\mathrm{W} / \mathrm{h}$, the function tends to zero so that the interpolation moves to the ASTM solution. The weighting given to each solution is shown in figure 12 . The $0.2 \mathrm{~h} / \mathrm{a}$ factor in the equation was found empirically. 


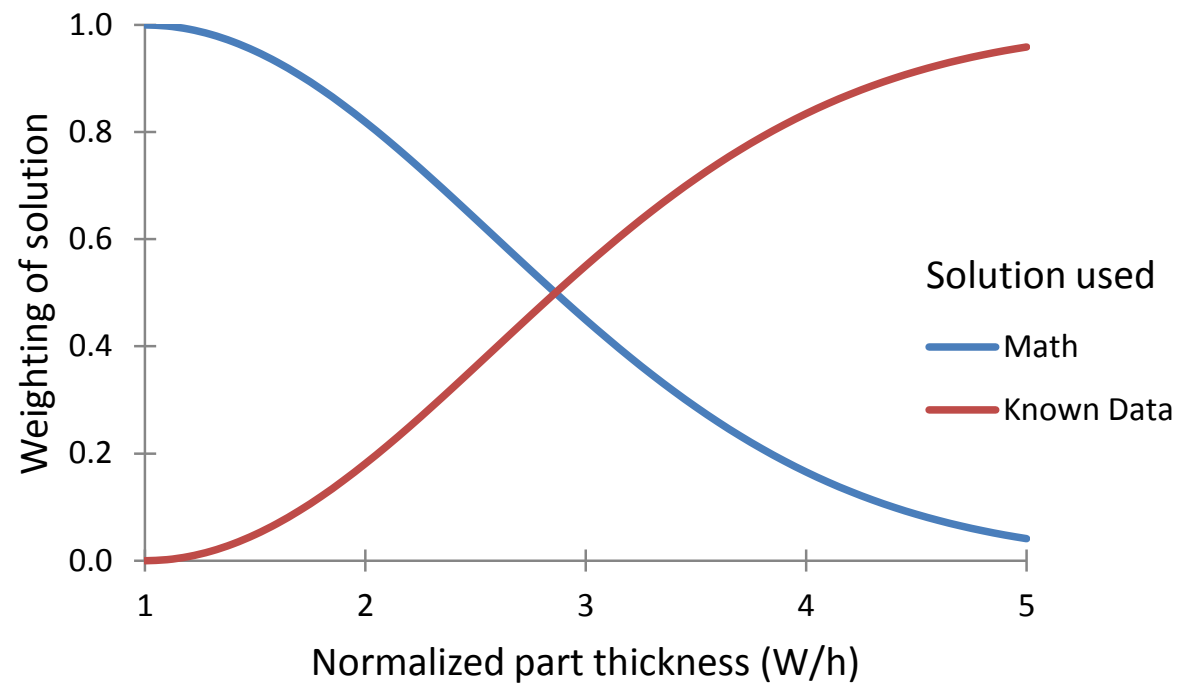

Figure 12: Showing weighting of mathematical solution and known thick case solution for interpolation function.

Figure 13 plots the calibration constant $\bar{a}$ in the same way as figure 11 , but with the additional interpolation function to the thick case. Looking at the graph of the calibration constants with the modified math solution and the interpolation function it is clear that the adjustments are effective in producing useable data.

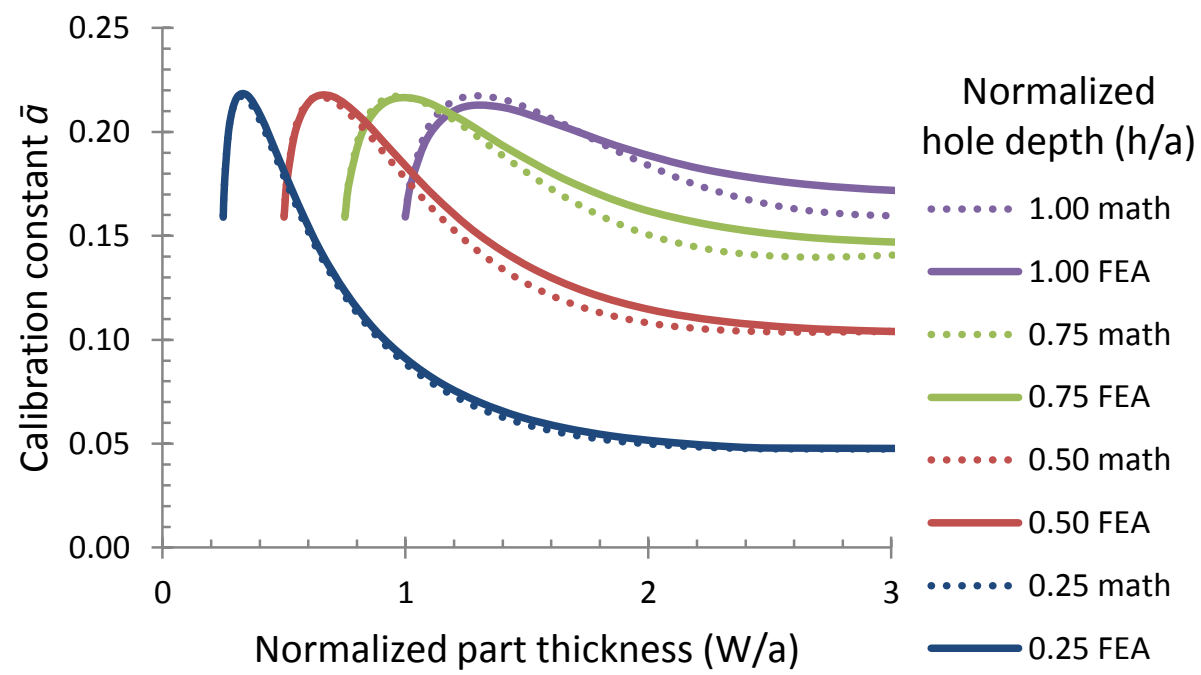

Figure 13: Comparing calibration constant $\bar{a}$ for mathematical solution and finite element solution with adjustment and interpolation. 
ESPI measurements have the advantage that they can measure either in plane displacements or out of plane displacements. The equations for the out of plane motion can also be used with the load distribution derived above. The equation for the vertical displacement as a function of radius shown previously is combined with the net moment acting on the outer ring.

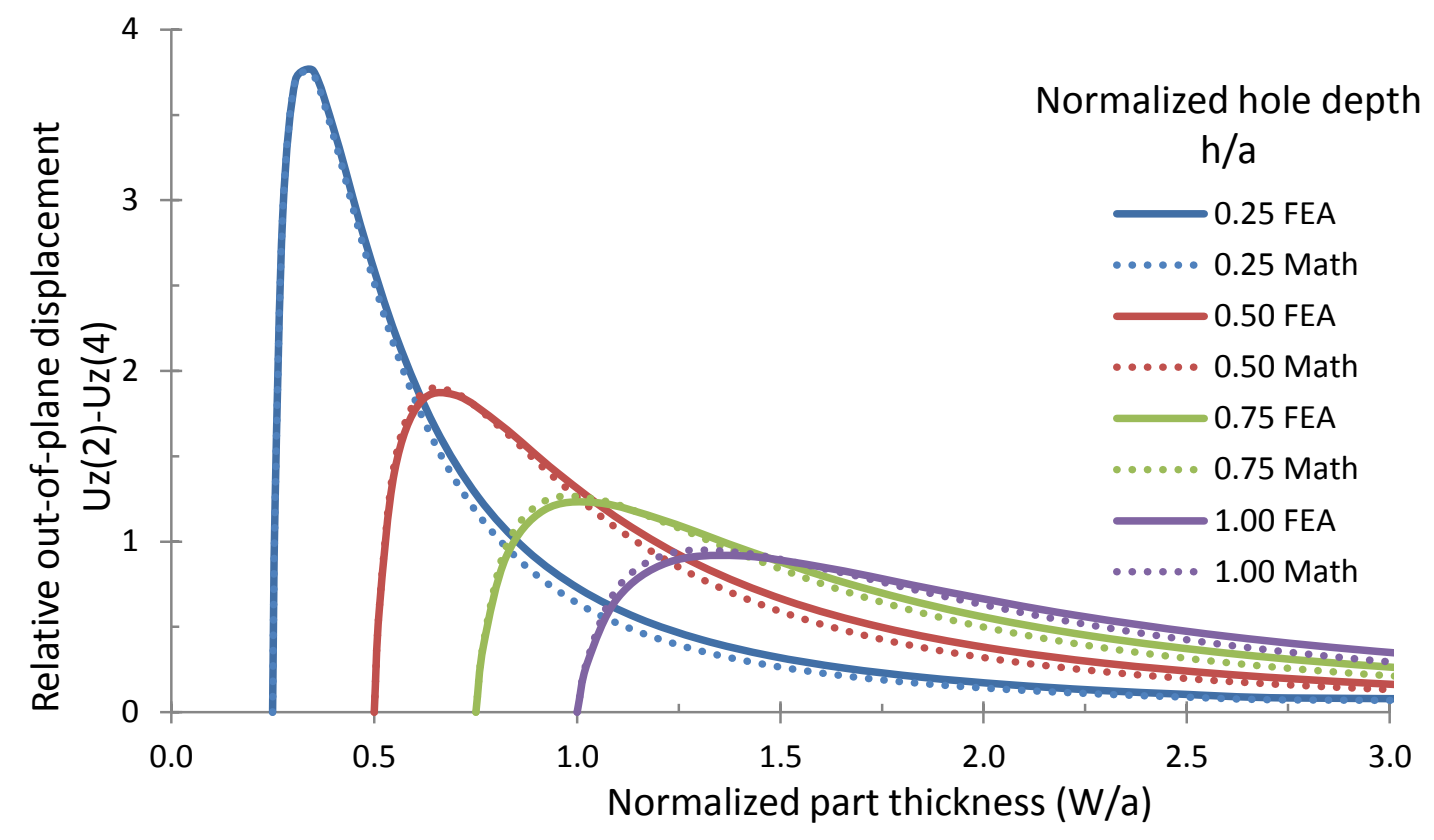

Figure 14: Out-of-plane relative displacements

The out-of-plane bending case shown in Fig.14 needs no interpolation to the thick case because as the part gets thicker the stiffness increases such that the out of plane bending tends to zero. The intermediate case will be sensitive for thicknesses modestly greater than the thin case but cannot be used for very thick specimens.

\subsubsection{Equations for Case B}

The second case needed to analyse hole drilling data is the shear stress case. This case corresponds to the calibration constant $b$. The equations for the B case are derived in a similar fashion as those for the A case. There is an additional dependency because these equations are no longer axi-symmetric; the equations all vary with the sine or cosine of $2 \theta$. The load distribution is the same as in the A case but with different values for the stiffness's of the inner disk and outer ring. The new values are: 


$$
\begin{array}{ll}
k_{1}=\frac{(W-h) E}{1+v} & k_{2}=\frac{W E}{3-v} \\
K_{1}=\frac{(W-h)^{3} E}{12(1+v)} & K_{2}=\frac{W^{3} E}{4(1+v)}
\end{array}
$$

The net horizontal force $\mathrm{C}$ and moment $\mathrm{Q}$ can then be calculated by substituting the new ' $\mathrm{k}$ ' values into the previously derived equations.

$$
\begin{aligned}
& Q=S\left[\frac{2 k_{1} h K_{2}+2 K_{2}\left(k_{1}+k_{2}\right)(W-H)}{k_{1} k_{2} h^{2}+4\left(k_{1}+k_{2}\right)\left(K_{1}+K_{2}\right)}\right] \\
& C=S\left[\frac{4 k_{2}\left(K_{1}+K_{2}\right)+k_{1} k_{2} h(W+h-H)}{4\left(k_{1}+k_{2}\right)\left(K_{1}+K_{2}\right)+k_{1} k_{2} h^{2}}\right]
\end{aligned}
$$

The load distribution equations may then be applied to the equations of motion for the outer ring.

For the $\mathrm{B}$ case the in-plane displacements are given by the equation for a thin plate with a hole with a pressure, $P$, applied at the inner edge. The derivation for this equation can be found in [2].

$$
\begin{array}{r}
U_{r}=\frac{P r}{E}\left[4 \frac{a^{2}}{r^{2}}-(1+v) \frac{a^{4}}{r^{4}}\right] \cos (2 \theta) \\
U_{\theta}=\frac{P r}{E}\left[2(1-v) \frac{a^{2}}{r^{2}}+(1+v) \frac{a^{4}}{r^{4}}\right] \sin (2 \theta)
\end{array}
$$

The equations bending of the outer ring for the $\mathrm{B}$ case were derived from the general plate solution laid out in [12]. The bending of the outer ring is an anticlastic bending case that forms a saddle shape.

The equation for a thin plate with no lateral load is

$$
D \nabla^{4} w=0
$$

Where

$$
\nabla^{2}=\frac{\partial^{2}}{\partial r^{2}}+\frac{1}{r} \frac{\partial}{\partial r}+\frac{1}{r^{2}} \frac{\partial^{2}}{\partial \theta^{2}}
$$

For the anticlastic case

$$
\frac{\partial^{2} w}{\partial r^{2}}=-w \cos (2 \theta)
$$


Using the general solution of form $w(r)=r^{n}$

$$
\left(\frac{d^{2}}{d r^{2}}+\frac{1}{r} \frac{d}{d r}-\frac{4}{r^{2}}\right)(n(n-1)+n-4) r^{n-2}=0
$$

Integrating this equation leads to

$$
(n+2)(n-2) n(n-4) r^{n-4}=0
$$

For a nontrivial solution, $\mathrm{n}=-2,0,2$ or 4

$$
W(r)=C_{1}+\frac{C_{2}}{r^{2}}+C_{3} r^{2}+C_{4} r^{4}
$$

The integration can be evaluated from here using the boundary conditions.

$$
\begin{array}{ll}
M_{r}(a)=M_{O} & Q(a)=\mathbf{0} \\
M_{r}(\infty)=\mathbf{0} & Q(\infty)=\mathbf{0}
\end{array}
$$

Using the general solutions from [12].

$$
\begin{aligned}
& M_{r}=-D\left(\frac{\partial^{2} w}{\partial r^{2}}+\frac{v}{r} \frac{\partial w}{\partial r}+\frac{v}{r^{2}} \frac{\partial^{2} w}{\partial \theta^{2}}\right) \\
& M_{\theta}=-D\left(\frac{1}{r^{2}} \frac{\partial^{2} w}{\partial \theta^{2}}+\frac{1}{r} \frac{\partial w}{\partial r}+v \frac{\partial^{2} w}{\partial r^{2}}\right) \\
& M_{r \theta}=-D(1-v)\left(\frac{1}{r} \frac{\partial^{2} w}{\partial r \partial \theta}-\frac{1}{r^{2}} \frac{\partial w}{\partial \theta}\right) \\
& Q_{r}=\frac{\partial M_{r}}{\partial r}+\frac{M_{r}-M_{\partial}}{r}+\frac{1}{r} \frac{\partial M_{r \theta}}{\partial \theta}
\end{aligned}
$$

For

$$
\begin{array}{ll}
W(r)=C_{1}+\frac{C_{2}}{r^{2}} & \frac{\partial W}{\partial r}=-2 \frac{C_{2}}{r^{3}} \\
\frac{\partial^{2} W}{\partial r^{2}}=6 \frac{C_{2}}{r^{4}} & \frac{\partial^{3} W}{\partial r^{3}}=-24 \frac{C_{2}}{r^{5}}
\end{array}
$$

Using the above equation sets, the constants of integration $\mathrm{C}_{1}$ and $\mathrm{C}_{2}$ can be solved for. 


$$
C_{1}=0 \quad C_{2}=-\frac{M_{o} a^{4}}{6 D(1-v)}
$$

Inserting this into the original equation gives the out of plane displacement as a function of radius and theta for a bi-harmonic moment applied at the hole edge.

$$
W(r, \theta)=\frac{2(1+v) a^{3}}{E W^{3} r^{2}} M_{o} \cos (2 \theta)
$$

Differentiating this in the radial direction gives radial slope.

$$
\varphi_{r}=\frac{\partial W}{\partial r}=-\frac{4(1+v)}{E W^{3}} \frac{a^{3}}{r^{3}} M_{o} \cos (2 \theta)
$$

Differentiating in the tangential direction gives tangential slope.

$$
\varphi_{\theta}=\frac{1}{r} \frac{\partial W}{\partial \theta}=-\frac{4(1+v)}{E W^{3}} \frac{a^{3}}{r^{3}} M_{o} \sin (2 \theta)
$$

As in the A case the total radial displacement is the sum of the in-plane and bending deformation.

$$
U_{r}(r, \theta)=U_{r}(\text { in plane })+\frac{W}{2} \varphi_{r}
$$

The B case also has a tangential displacement not seen in the A case. This can be calculated in the same way that the radial displacement is found.

$$
U_{\theta}(r, \theta)=U_{\theta}(\text { in plane })+\frac{W}{2} \varphi_{\theta}
$$

Substituting previous equations and the load distribution, Q and C, into these we find the radial and tangential displacement for the $\mathrm{B}$ case as a function of $r$ and $\theta$.

$$
\begin{gathered}
U_{r}(r, \theta)=\left(\frac{C r}{W a E}\left[4 \frac{a^{2}}{r^{2}}-(1+v) \frac{a^{4}}{r^{4}}\right]+\frac{W}{2} \frac{4(1+v)}{E W^{3}} \frac{a^{3}}{r^{3}} Q\right) \cos (2 \theta) \\
U_{\theta}(r, \theta)=\left(\frac{C r}{W a E}\left[2(1-v) \frac{a^{2}}{r^{2}}+(1+v) \frac{a^{4}}{r^{4}}\right]+\frac{W}{2} \frac{4(1+v)}{E W^{3}} \frac{a^{3}}{r^{3}} Q\right) \sin (2 \theta)
\end{gathered}
$$

Using the same procedure outlined above for the A case, the displacement data is used to compute the calibration coefficient $Ђ$. These results are then plotted the same way as the A case and shown in figure 15. 


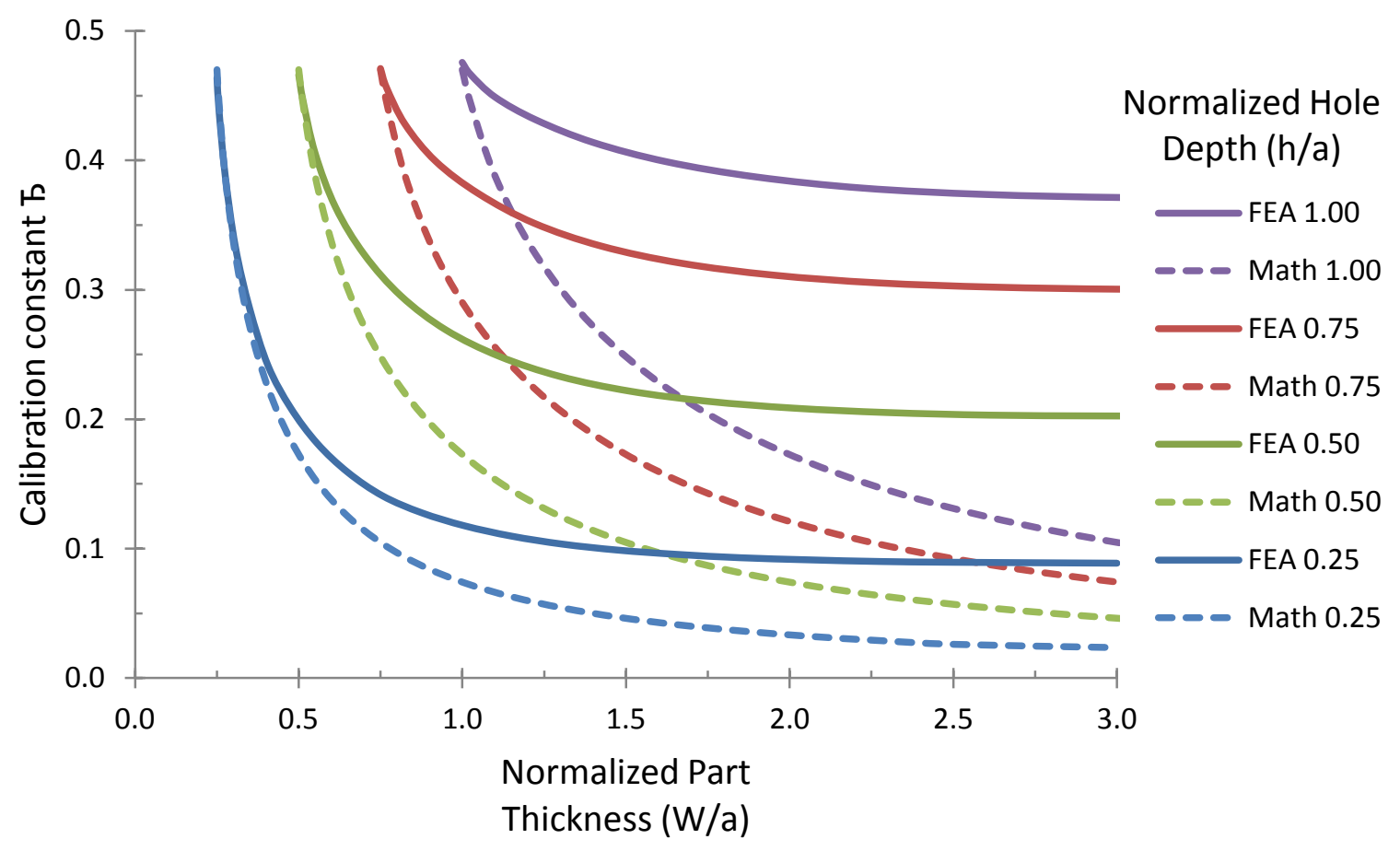

Figure 15: Calibration constant $\mathrm{5}$ for various normalized hole depths as relative part thickness increases.

The above graph shows significant discrepancies between the mathematical solution and the known solution. This is caused by the deflection at the boundary between the outer ring and the inner disk. This deflection is more significant than the $\mathrm{A}$ case because the stress concentration factor for the B case is double that of the A case. The boundary between the inner disk and outer ring staying linear is one of the initial assumptions. The fact that this is not the case, as shown in figure 16, means that the equations are no longer valid. Adjustments to the mathematical solution are proposed to account for this discrepancy. 


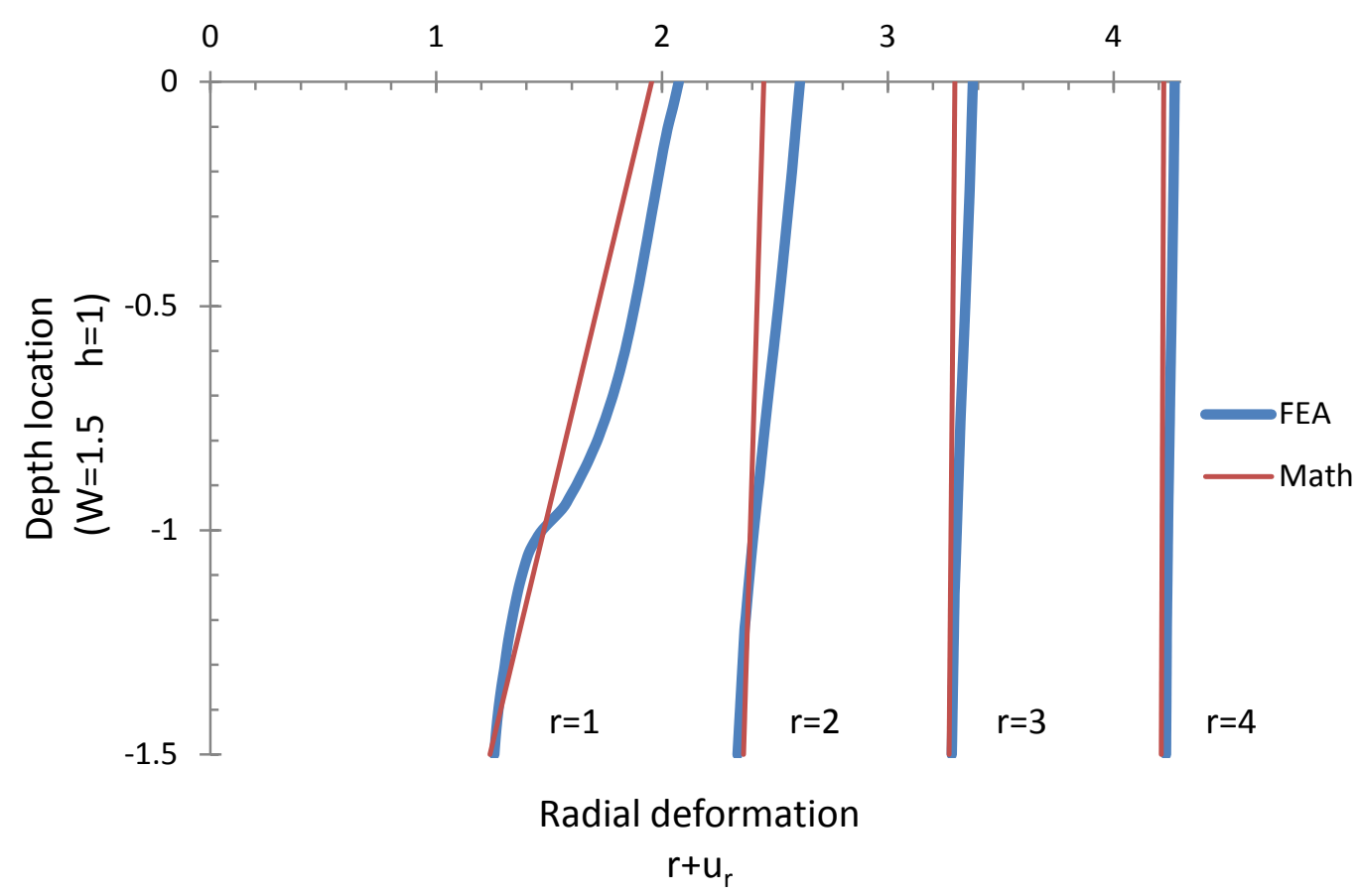

Figure 16: Displacements through part thickness at various radiuses for case B comparing finite element analysis data to math solution.

(Displacements are plotted $r+u_{r}$ for clarity)

The adjustments were selected so that a minimum change to the equations was made while still getting good agreement. These adjustments were found empirically by examining the finite element data. The surface deformations over a wide range of thicknesses and hole depths were used as well as the through thickness information.

$$
\begin{gathered}
k_{1}=\frac{(W-h) E}{(1+v)} \quad k_{2}=\frac{W E}{(2-v)} \\
K_{1}=\frac{(W-h)^{3} E}{12(1+v)} \quad K_{2}=\frac{W^{3} E}{4(1+v)} \\
U_{r}(r, \theta)=\left(\frac{C r}{W a E}\left[4 \frac{a^{2}}{r^{2}}-(1+v) \frac{a^{4}}{r^{4}}\right]+\frac{W}{2} \frac{(3+v) 4(1+v)}{E W^{2}} \frac{a^{3}}{r^{3}} Q\right) \cos (2 \theta) \\
U_{\theta}(r, \theta)=\left(\frac{C r}{W a E}\left[2(1-v) \frac{a^{2}}{r^{2}}+(1+v) \frac{a^{4}}{r^{4}}\right]+\frac{W}{2} \frac{(3+v) 4(1+v)}{E W^{2}} \frac{a^{3}}{r^{3}} Q\right) \sin (2 \theta)
\end{gathered}
$$

The area at the hole boundary still does not agree but this is admissible due to the fact that the deformations are measured further away from the edge of the hole for both ESPI and strain gauge measurements. Measuring away from the edge of the hole also helps to avoid the local damage that may be caused during the drilling process. The displacements from the modified mathematical solution are displayed in figure 17. 


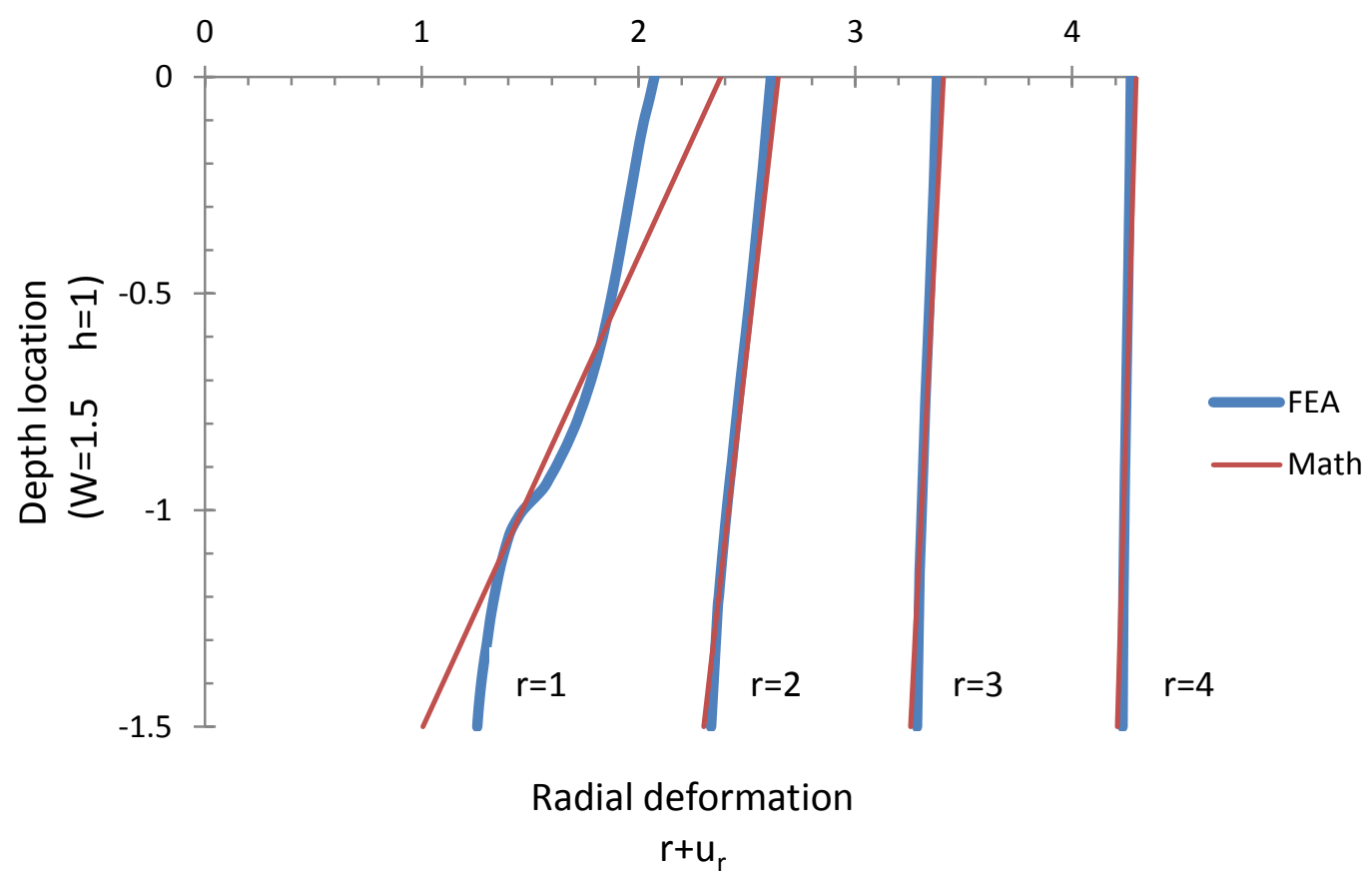

Figure 17: Displacements through part thickness at various radiuses for case B comparing finite element analysis data to the modified math solution (equation 69-71). (Displacements are plotted $r+u_{r}$ for clarity)

These new equations are then used to reproduce the previous graph showing the variation of the calibration constant $万$ with respect to part thickness. 


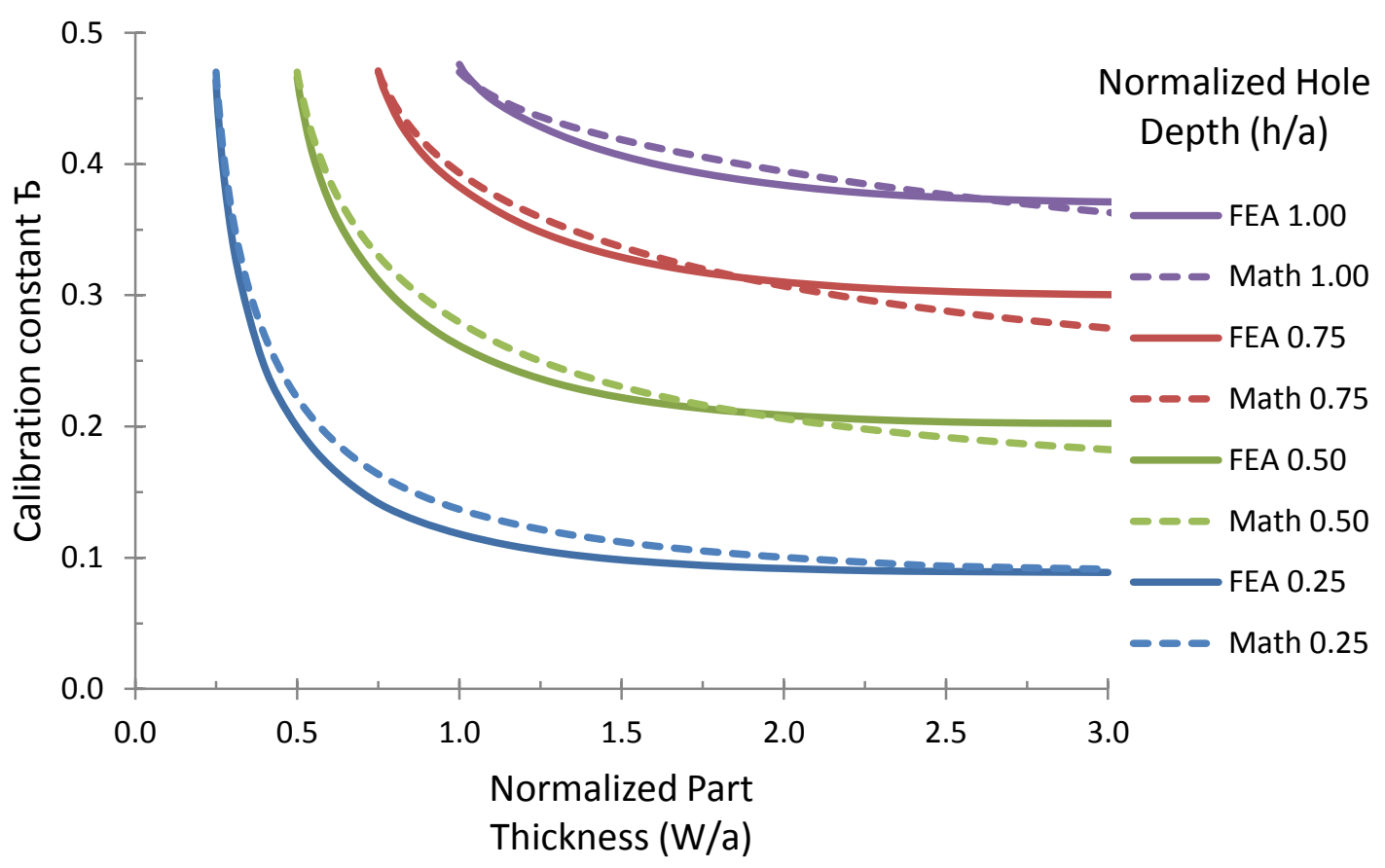

Figure 18: Calibration constant Ђ for various hole depths as relative part thickness increases with adjustments to math.

The results in figure 18 compare much better than with the unadjusted math. The interpolation disused in the previous chapter is now applied in the same way that it was for the A case. The resulting graph is presented in figure 19 and the out of plane graph is shown in figure 20. 


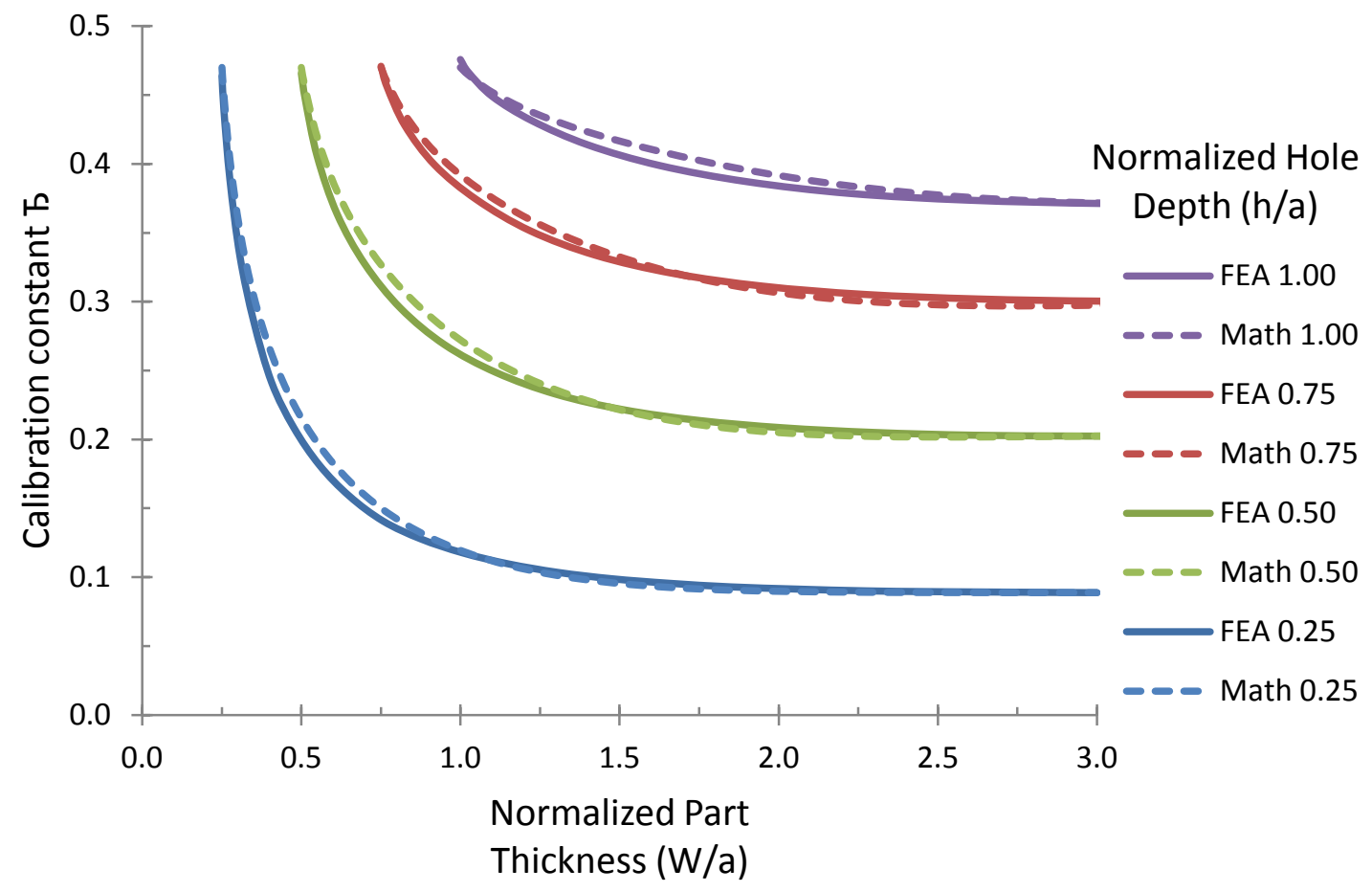

Figure 19: Calibration constant Ђ for various hole depths as relative part thickness increases with adjustments to math and thick case interpolated.

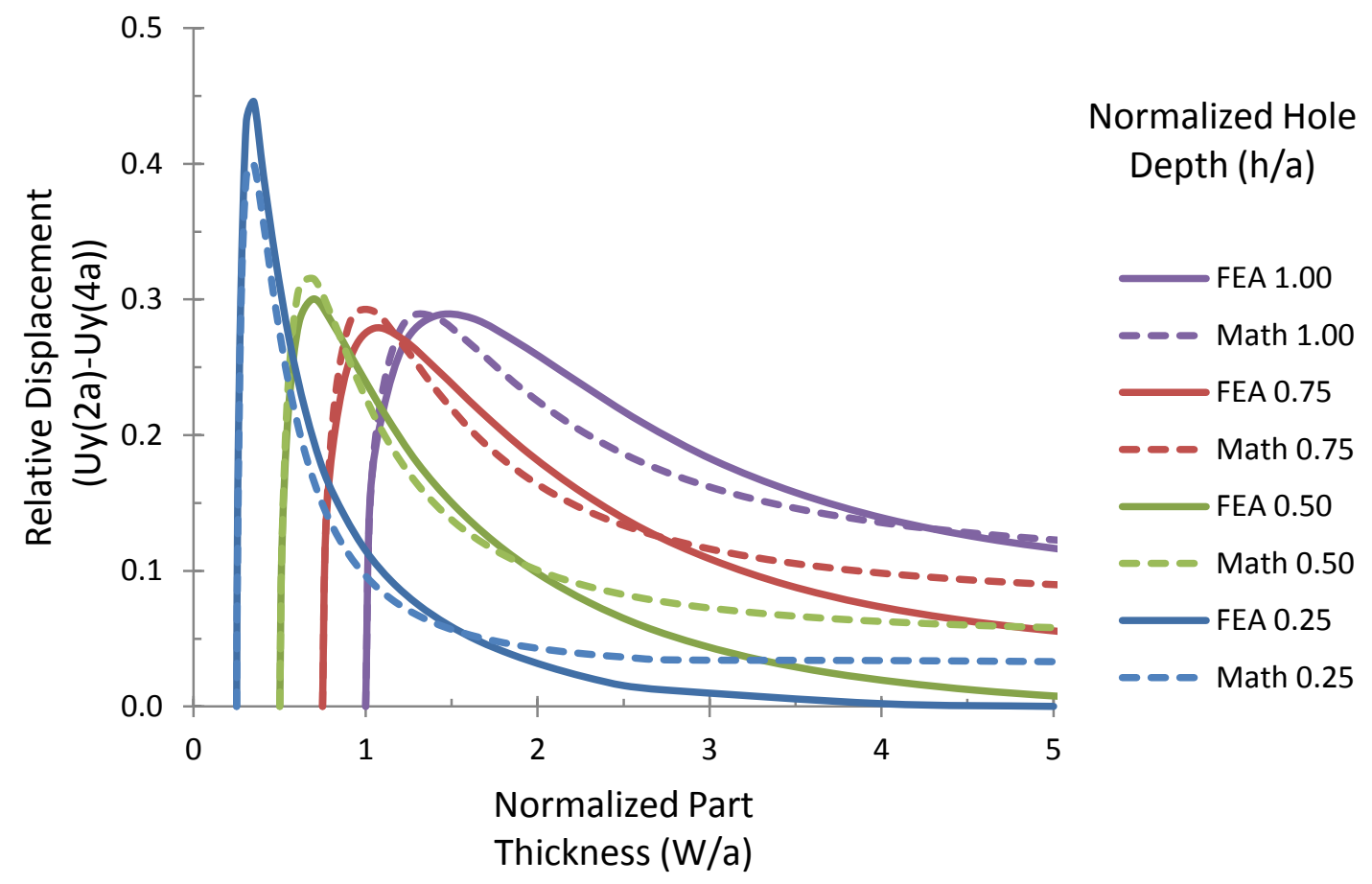

Figure 20: Relative out-of-plane displacements for various hole depths as relative part thickness increases. 


\subsubsection{Stress Profiling}

In residually stressed parts, the stresses are often not uniform through the thickness of the part. The stress profile describes how the stresses vary with respect to depth. The stress profile is often of specific interest because processes such as shot peening or other surface treatments create stresses whose magnitude changes significantly with depth.
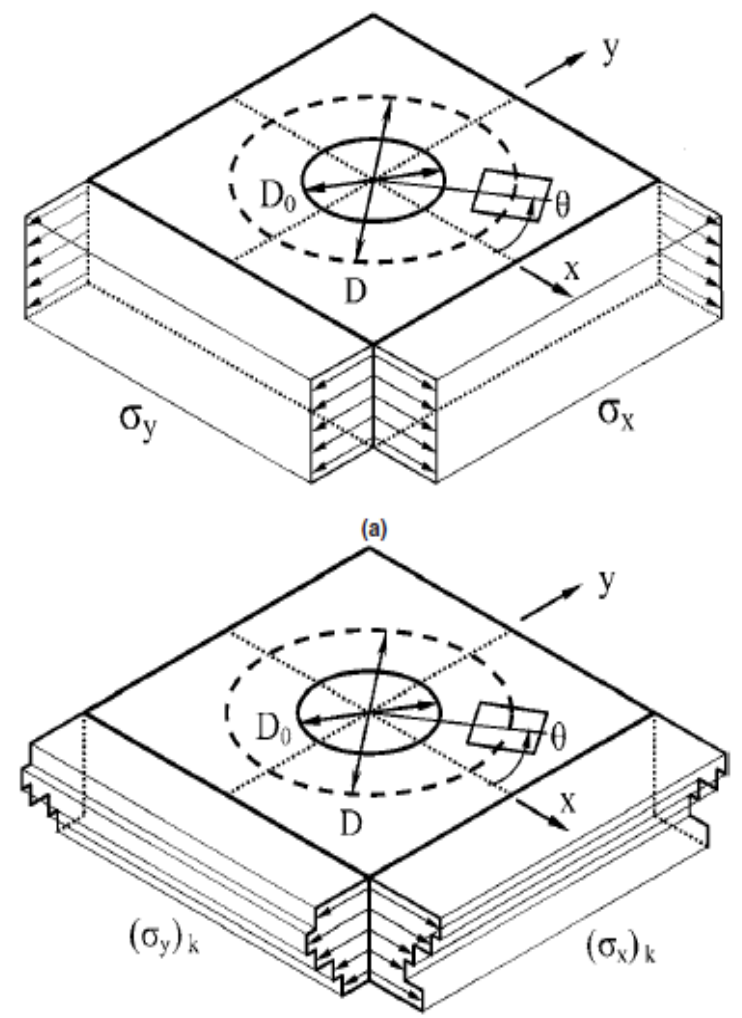

(b)

Figure 21: Stress profiles for average stress measurements (a), and stress profiling measurements (b) (adapted from [13])

The stress profile is measured by drilling incrementally into the workpiece. The strain around the hole is measured after each incremental depth is drilled. The stresses are then calculated at each step. Several methods for determining the stress profile exist in the literature including the Incremental Strain Method, Average Stress Method, Power Series Method and Integral Method. A good overview of the various methods is given in [13] and [14]. The Integral Method for determining stress profiles is the most accurate. The stress at each depth can be calculated from equation 72 given below. For clarity the A case is discussed here, but the B case can be analyzed similarly by simply substituting the corresponding $\mathrm{B}$ values for the A values here. 


$$
\sum_{j=1}^{j=i} \overline{\mathrm{a}}_{i j} \sigma_{j}=\frac{E}{1+v} \varepsilon_{i} \quad 1 \leq j \leq i \leq n
$$

Where $\varepsilon_{\mathrm{i}}=$ Measured strain relaxation after the $i$ th hole depth increment $\sigma_{\mathrm{j}}=$ Equivalent uniform stress within the $j$ th hole depth increment

$\overline{\mathrm{a}}_{\mathrm{ij}}=$ Strain relaxation due to a unit stress within increment $j$ of a hole $i$ increments deep which can be calculated from equation 41

$\mathrm{n}=$ total number of hole depth increments
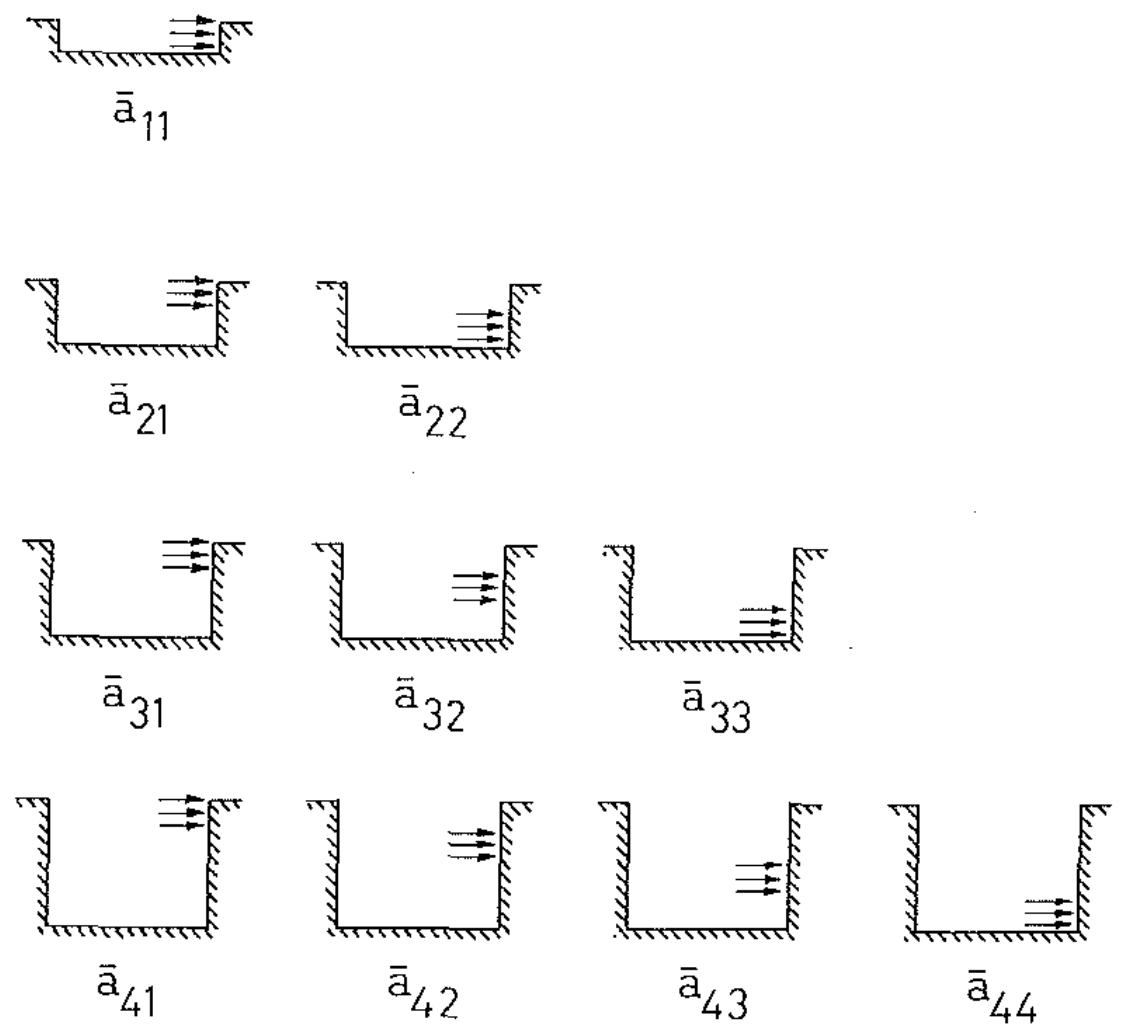

Figure 22: Physical interpretation of $\overline{\mathrm{a}}_{i j}$ from equation 72 (adapted from [13])

Having a mathematical solution that can calculate $\bar{a}_{i j}$ and $\bar{b}_{i j}$ for any part thickness allows the application of stress profiling methods to the thin and intermediate thickness workpiece. This significantly broadens the application of the hole drilling method as measuring stress profiles for thin workpieces is often quite desirable. 


\section{Chapter 3 Application of Results}

\subsection{Experimental Validation}

In order to validate the equations derived above, the equations are compared to physical tests. Test specimens were prepared so as to have minimal residual stresses. These specimens were then bent to a known curvature to impart a known stress state. The specimens were then measured with the newly derived technique and results compared to the expected values.

\subsection{Experimental Setup}

The experimental setup is shown in figure 23. All elements in the experimental setup are mounted on an optical table which can be seen in the background. This allows everything to be rigidly fixed to the base. The optical table then sits on air bearings to minimize the vibrations from the surroundings and the entire setup is enclosed in a housing to prevent air currents or dust from disturbing the setup.

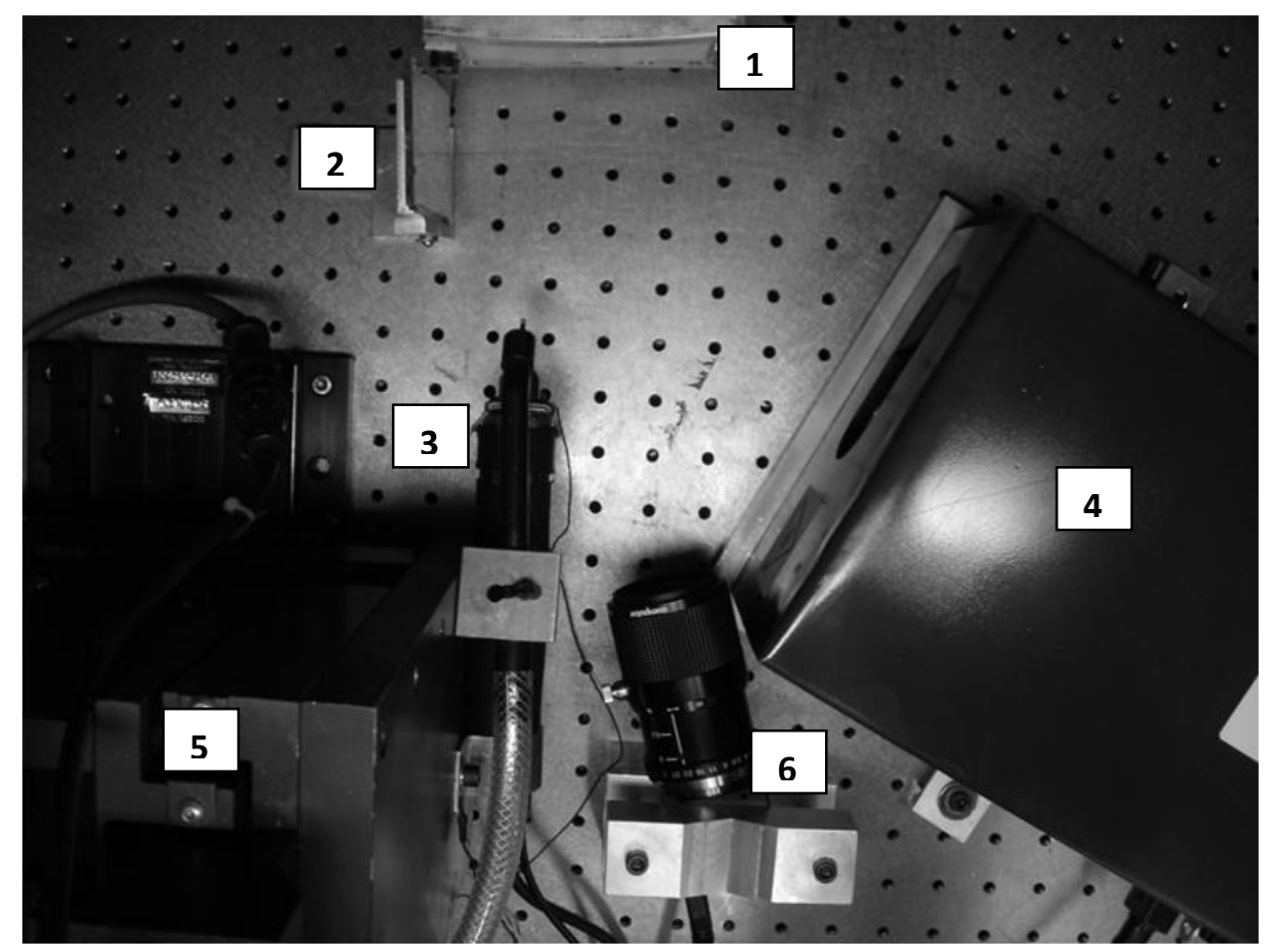

Figure 23: Layout of experimental setup. 1 Specimen, 2 Reference beam mirror, 3 Drill, 4 ESPI box, 5 Drill stage, 6 CCD Camera 
The specimen is held in the specimen holder figure 23 object 1 and figure 24 . The specimen holder consists of a base with slots for bolts to mount it to the optical table and a machined curve on the front with a section milled out. The specimen is bolted to the curved front of the specimen holder. The curvature bends the specimen to a known radius and this imparts calculable stresses. These stresses can be calculated using equation 73 and figure 25 . The milled out area allows the specimen to be free from behind so the thin case assumptions hold true. For the specimen holder used in this experiment the bend radius, $r_{\text {curve }}$, is $561.8 \mathrm{~mm}$.

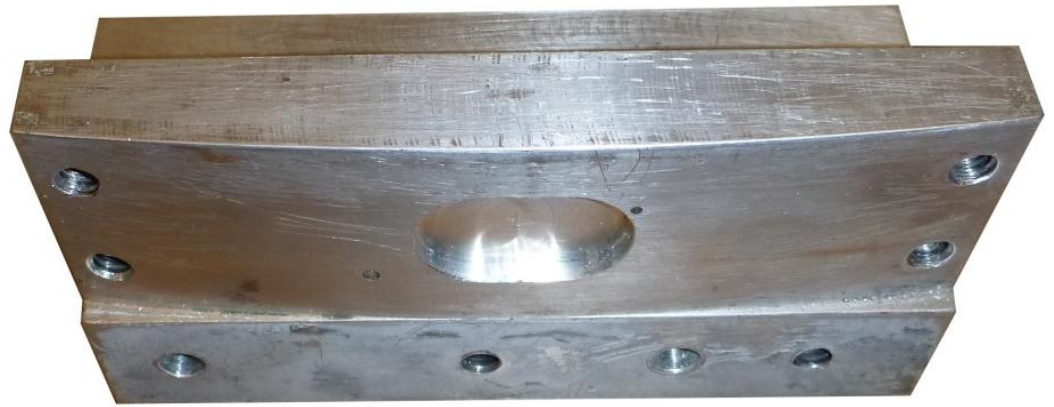

Figure 24: Specimen holder. Note the curvature on the front where the specimen mount and the slot machined out to allow for the thin case assumption.

$$
\sigma=\frac{E}{r_{\text {curve }}} * \mathrm{Y}
$$

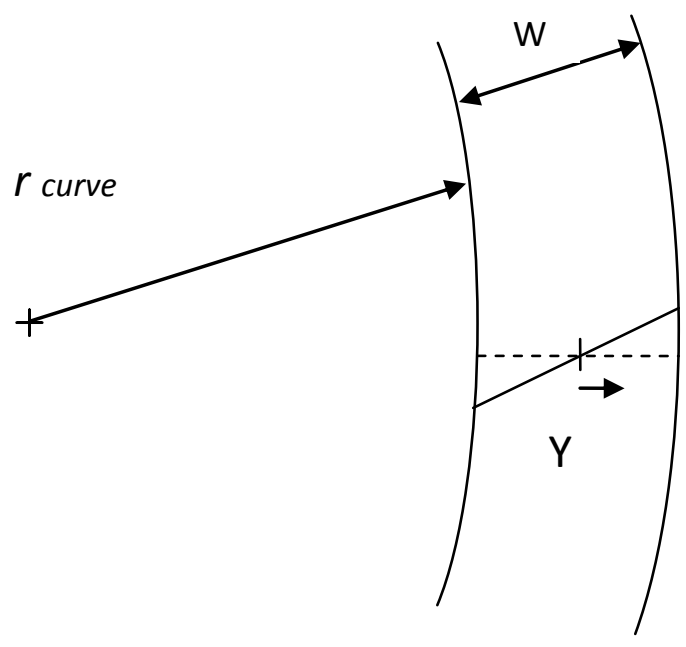

Figure 25: Bending of specimen showing linearly varying stresses through the thickness of the part 
A standard test specimen is shown in figure 26. Proper specimen preparation is paramount to obtaining good results. The specimen must be free of any initial residual stresses. Annealing the specimen is not an option as this weakens the material too much and plastic yielding occurs. Specimen are cut out of 6061 T-6 aluminum and are skimmed with a fly cutter. Skimming the specimen removes ten to twenty thousandths of an inch of material off each side. High levels of residual stresses are often present in this area from the rolling process used to make the sheet metal. The skimming operation imparts minimal stresses back into the material because fly cutters provide single point contact cutting and low thermal gradients. As a final step, the surface of the specimen is painted with a thin layer of white paint. This provides the needed surface roughness for ESPI speckles and reduces glare giving a more evenly illuminated surface.

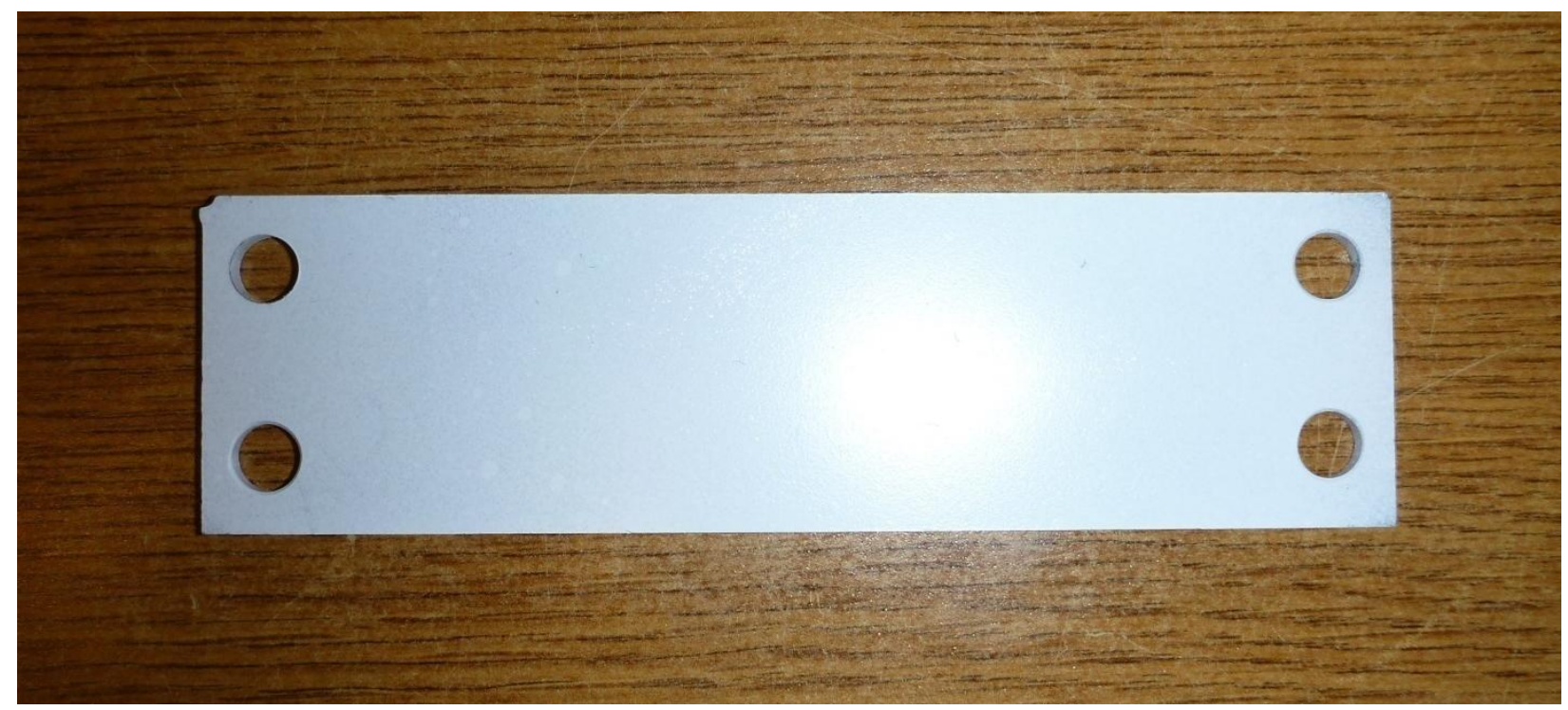

Figure 26: Specimen. This specimen has been skimmed to remove the surface stresses and painted with a thin coat of white paint.

The surface of the part is illuminated by a custom built ESPI setup, figure 23 object 4. This provides the interference pattern and phase stepping needed for the measurement tecnique, and will be discussed later.

The mirror noted by object 2 in figure 23 is needed to reflect the reference beam back onto the specimen as shown in figure 27. Having the two beams shine on the specimen at these angles produces a sensitivity vector in the $\mathrm{x}$ plane of the specemine. From this overhead view, that would be horizontal on the surface of the part. This means that the displacements in the $\mathrm{X}$ direction are the only diplacements measured directly. 


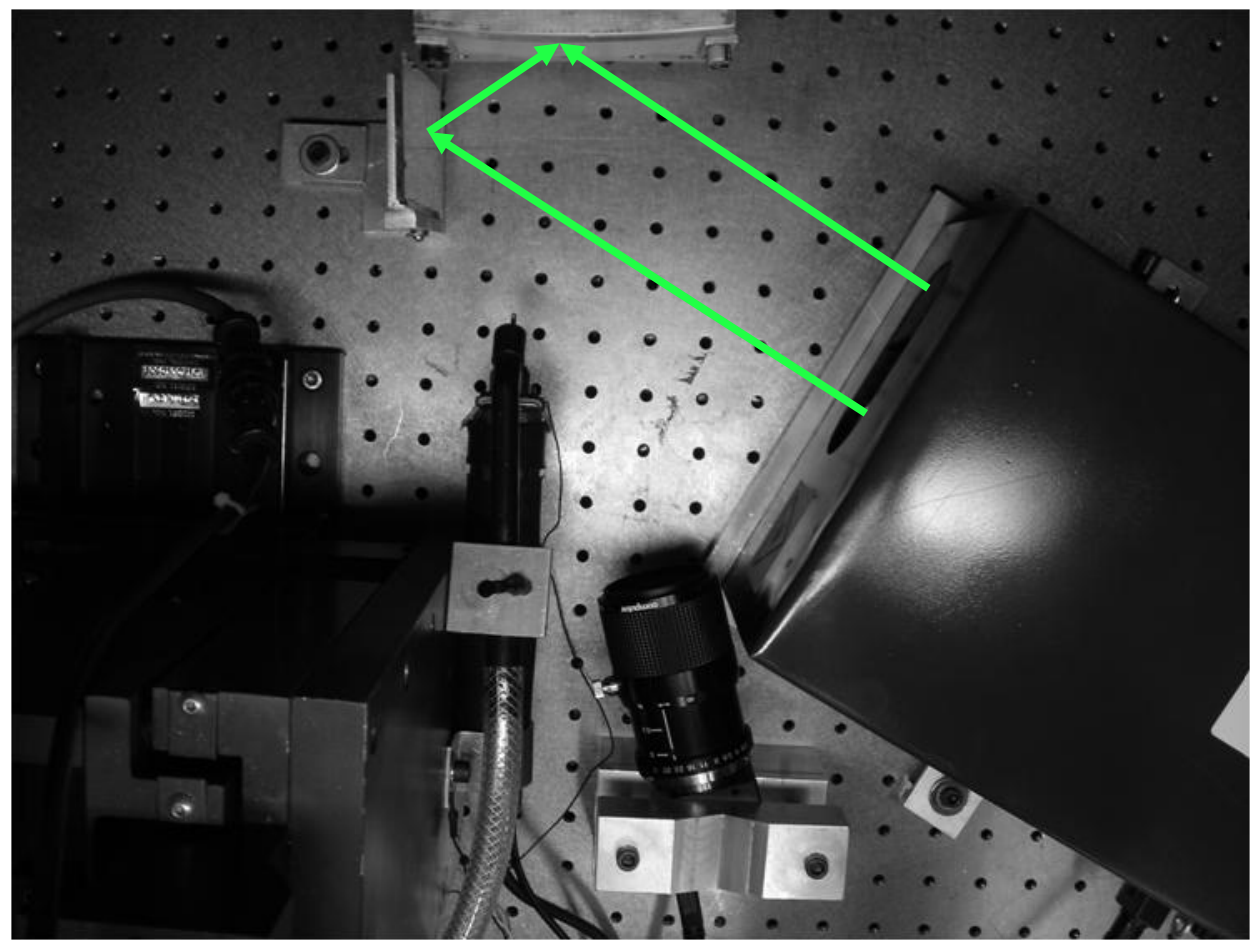

Figure 27: Path layout of object beam (right) and reference beam (left) from ESPI box to specimen holder.

The speckle pattern is imaged by a camera, figure 23 object 6 . The image is then stored on the computer for computation.

The drill, figure 23 object 3, is a high speed drill. Using a high speed drill helps to minimize stresses induced by the drilling process. A vacuum tube is attached to the head of the drill in an effort to capture chips as they are ejected. After the hole is drilled the part is lightly blown with compressed air which helps to remove any of the chips that may have landed on the part surface.

The drill is actuated by a three axis stage, figure 23 object 5 , which is computer controlled. This stage is accurate to plus or minus twenty thousandths of a millimetre. This allows the depth of cut to be controlled accurately and also ensures that subsequent depth steps are positioned concentrically.

The drill is fitted with an electrical contact sensor. This senses when the metal drill comes into contact with the part surface. This is ensures that the surface is correctly known so that the first depth step is the correct depth. Using electrical contact to sense the part surface also allows the thin layer of paint to be drilled through and the actual surface detected. 


\subsection{Electronic Speckle Patten Interferometry}

The experimental setup uses Electronic Speckle Pattern Interferometry (ESPI) to measure surface displacements as a hole is drilled in a specimen. The stresses in the specimen are known and compared to the measured values to determine the accuracy of the method.

The ESPI box was designed and fabricated specifically for this experiment. The design was focused in providing maximum stiffness to all parts, compactness of space and reliability. This design and fabrication represented significant investment of time and energy and a significant contribution to the lab. Stiffness of each component is critical as the ESPI method relies on all components remaining stable and even variations on the order of tens of nano-meters is enough to disrupt the measurement. The lenses need to be adjustable so the beams can be made parallel but rigid as well posing challenging design constraints. Compactness of space for the entire setup is desirable as the workspace is fairly constrained already. Reliability and ease of trouble shooting is important to the design for future use. The setup should be easily used and maintained for many future experiments. The entire circuitry is fuse protected and several status LEDs alert the user to potential problems and the current state of the device. Previous iterations did not include this feature and caused problems as the laser was overheating and becoming unstable. This was causing poor measurement quality, but the variations in laser intensity were not noticeable to the naked eye, making the problem hard to trace. These LEDs make future similar problems easy to identify and fix should they arrive.

The ESPI setup is pictured in figure 28 and the beam path is illustrated in figure 29. The apparatus consists of a laser that passes through a beam expander and a cylindrical lens. The purpose of the cylindrical lens is to create a uniform intensity distribution on the surface of the part knowing that the beam will impinge obliquely. The beam then travels through a series of mirrors and a beam splitter that diverges the beam into two paths. One beam path travels directly out the front of the apparatus, while the other reflects off a piezo mounted mirror before traveling out the front parallel to the first beam. Just before the beams travel out the front of the apparatus they go through a final lens to collimate the beam. The final result is two parallel collimated beams, one of which can be stepped by the piezo. 


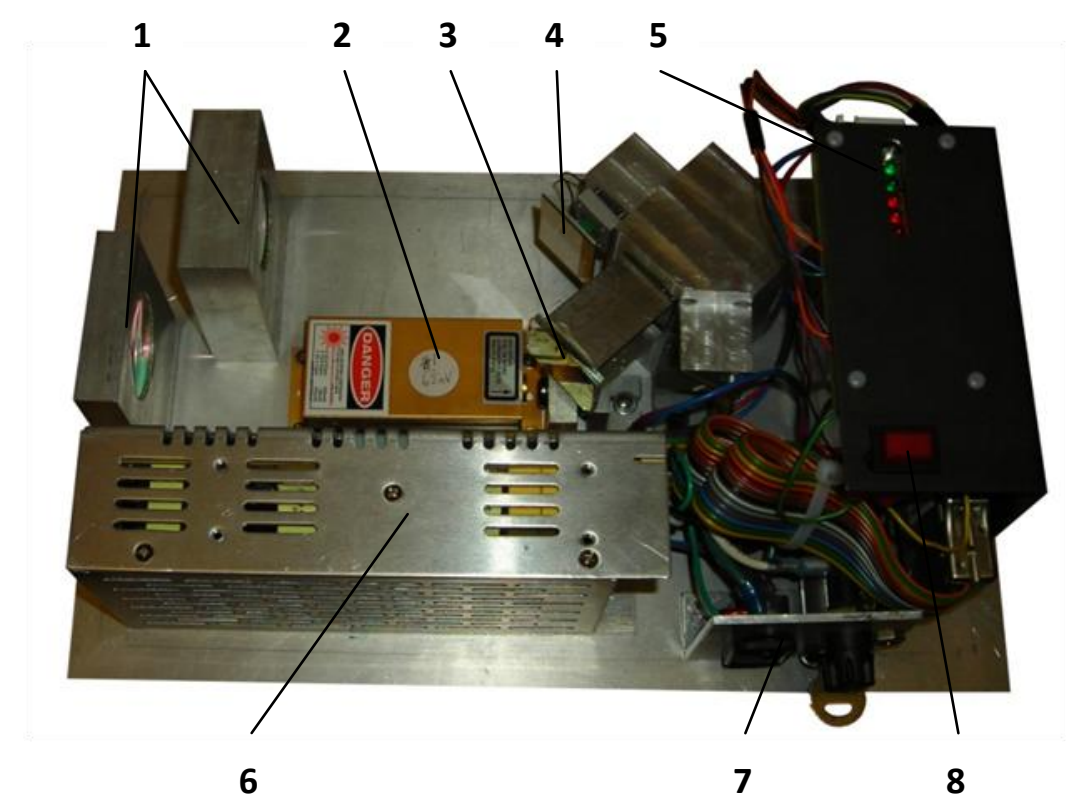

Figure 28: Layout inside ESPI box. 1 Collimating mirrors, 2 Laser, 3 Beam splitter, 4 Piezo stepped mirror, 5 Status LEDs, 6 Power supply, 7 Power, fuse protection and lock out key, 8 Power button

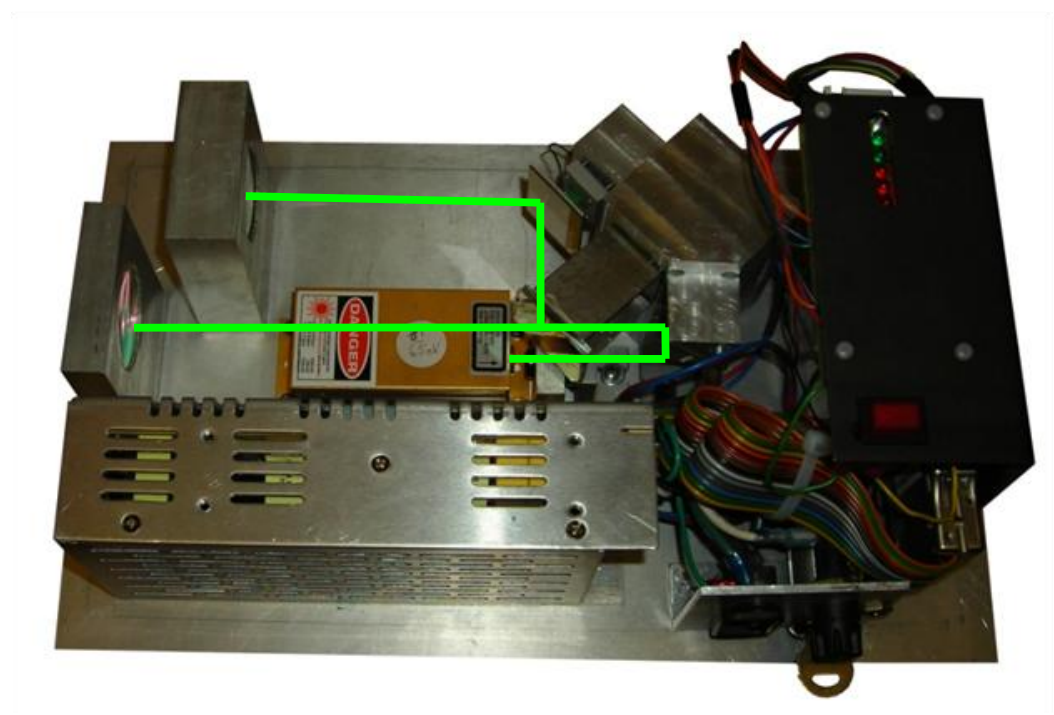

Figure 29: Beam path of the ESPI box.

Electronic speckle pattern interferometry is an optical method of measuring surface deformation [15]. ESPI relies on the interference of two coherent beams. Various beam arrangements can be made for measuring deformations either in-plane or out-of-plane. The setup used in this research measures in-plane displacements. When 
the two beams combine on the surface of the part, the light scatters off the roughness of the part and the constructive or destructive interference of the light creates speckles. The interference of the beams due to the surface roughness is shown in figure 30 . The speckles in this method are said to be subjective because their size depends on the aperture of the lens that they are viewed by.

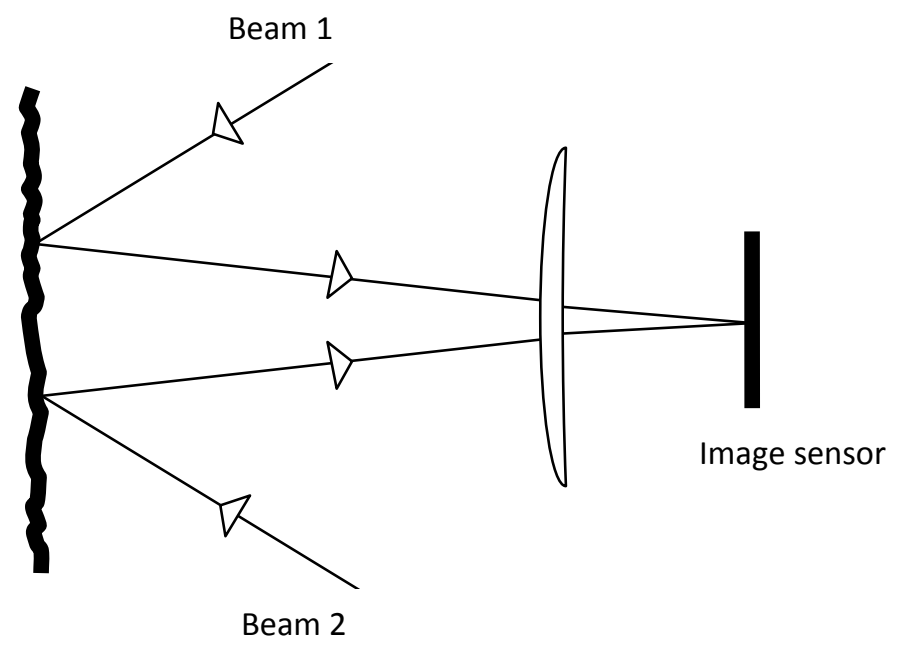

Figure 30: Ray diagram for ESPI speckles. Showing two beams reflecting off rough surface and resulting interference being imaged by camera.

The ESPI method uses two sets of four images, one set taken before the hole is drilled, figure 31, and one set taken after the hole is drilled, figure 32. Each one of the four images corresponds to the phase step of 90 degrees. 

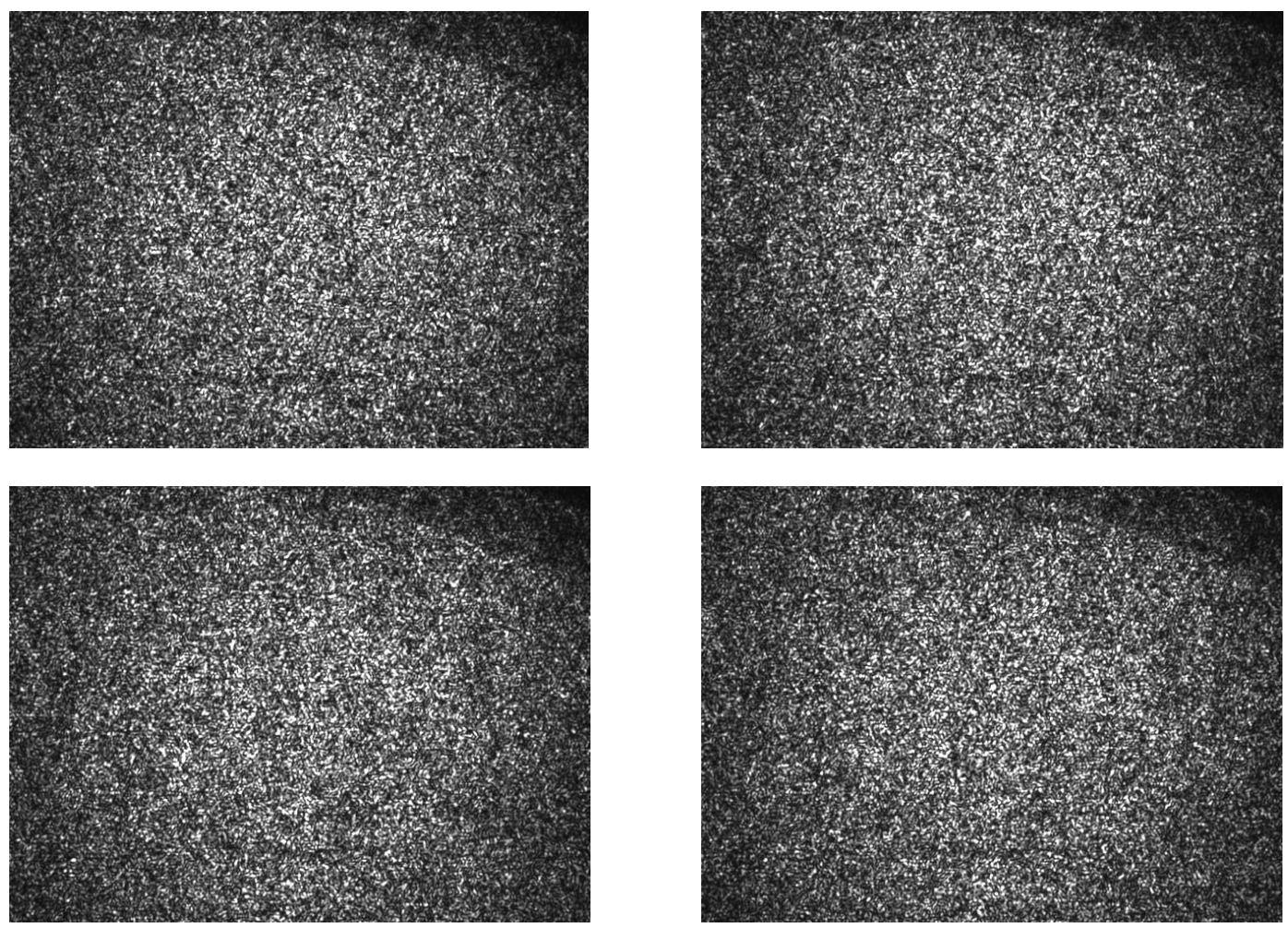

Figure 31: Set of four reference images for ESPI measurement. Images correspond to phase steps 0, 90, 180 and 270 . 

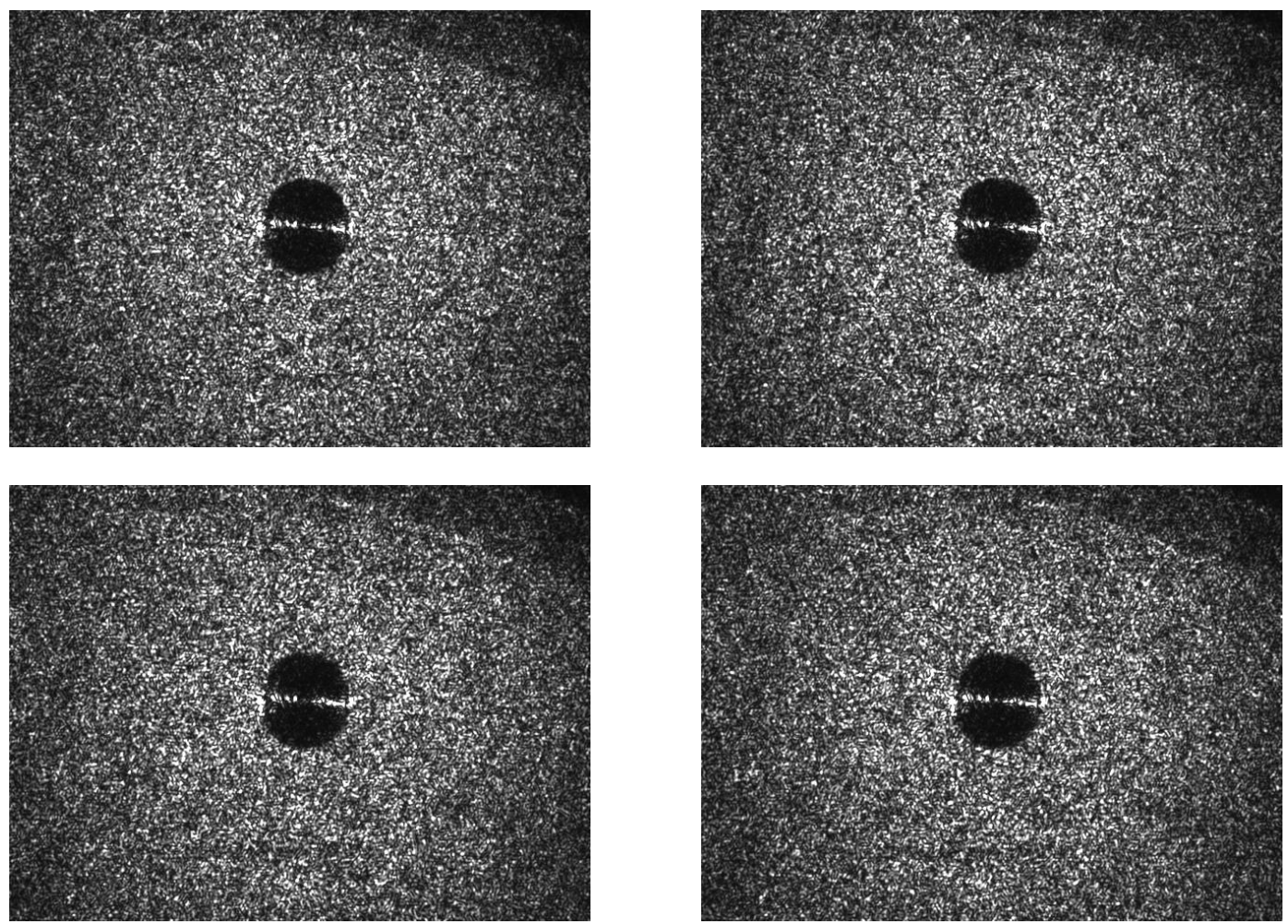

Figure 32: Set of four measurement images for ESPI measurement. Images correspond to phase steps 0, 90, 180 and 270. Notice the hole in the center of the image.

The intensity of each pixel, $I$, is a function of the two beams, $\mathrm{I}_{\mathrm{a}}$ and $\mathrm{I}_{\mathrm{b}}$, and their relative phase shift, $\phi_{\mathrm{R}}-\phi$. If they are in phase they will combine constructively, and if they are out of phase they will cancel each other out. The intensity of a pixel for each phase step is shown below.

$$
\begin{gathered}
I(0)=I_{a}{ }^{2}+I_{b}{ }^{2}+2 a b \cos \left(\phi_{R}-\phi\right) \\
I(90)=I_{a}{ }^{2}+I_{b}{ }^{2}+2 a b \sin \left(\phi_{R}-\phi\right) \\
I(180)=I_{a}{ }^{2}+I_{b}{ }^{2}-2 a b \cos \left(\phi_{R}-\phi\right) \\
I(270)=I_{a}{ }^{2}+I_{b}{ }^{2}-2 a b \sin \left(\phi_{R}-\phi\right)
\end{gathered}
$$

These four equations can be combined to solve for the relative phase change using the following equation. This is demonstrated visually in figure 33 .

$$
\phi_{R}-\phi=\arctan \left(\frac{I(90)-I(270)}{I(0)-I(180)}\right)
$$

This phase change can then be used to find the surface displacement. 


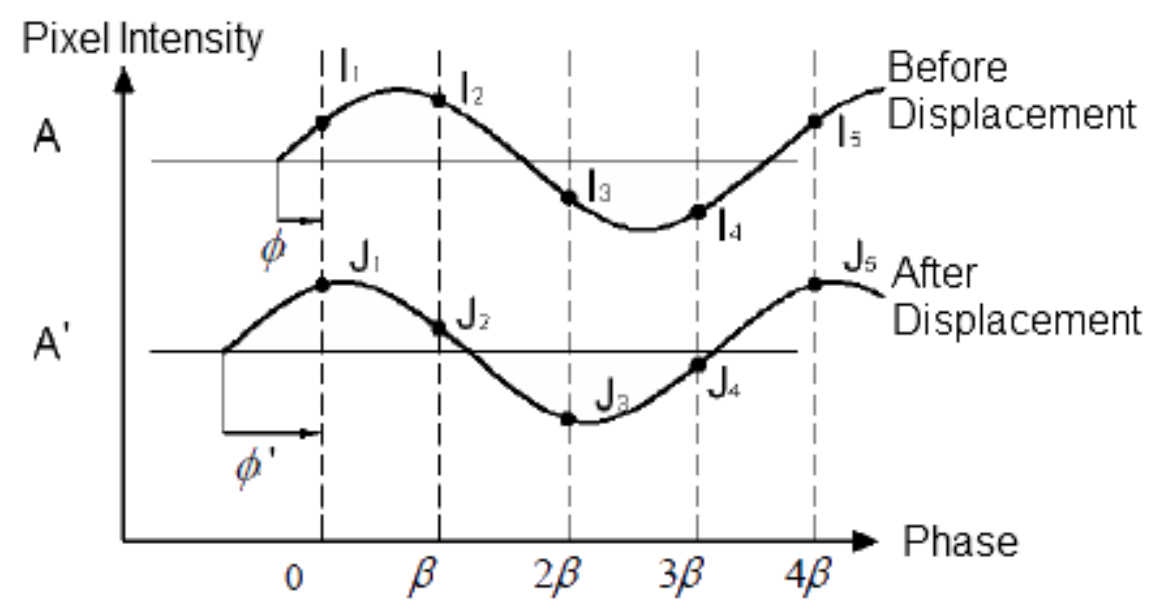

Figure 33: Using phase stepping and intensity data to compute relative phase change. Adapted from [16].

Plotting the relative phase change at each pixel creates an image like the one in figure 34. This is known as a fringe image. The fringe image can be converted into a displacement image knowing that each variation from light to dark is one quarter wavelength, which for the green laser used in this experiment is $133 \mathrm{~nm}$. This gives an idea of the accuracy that this method has. Due to the minute scale that these deflections are measured, great care must be taken. 


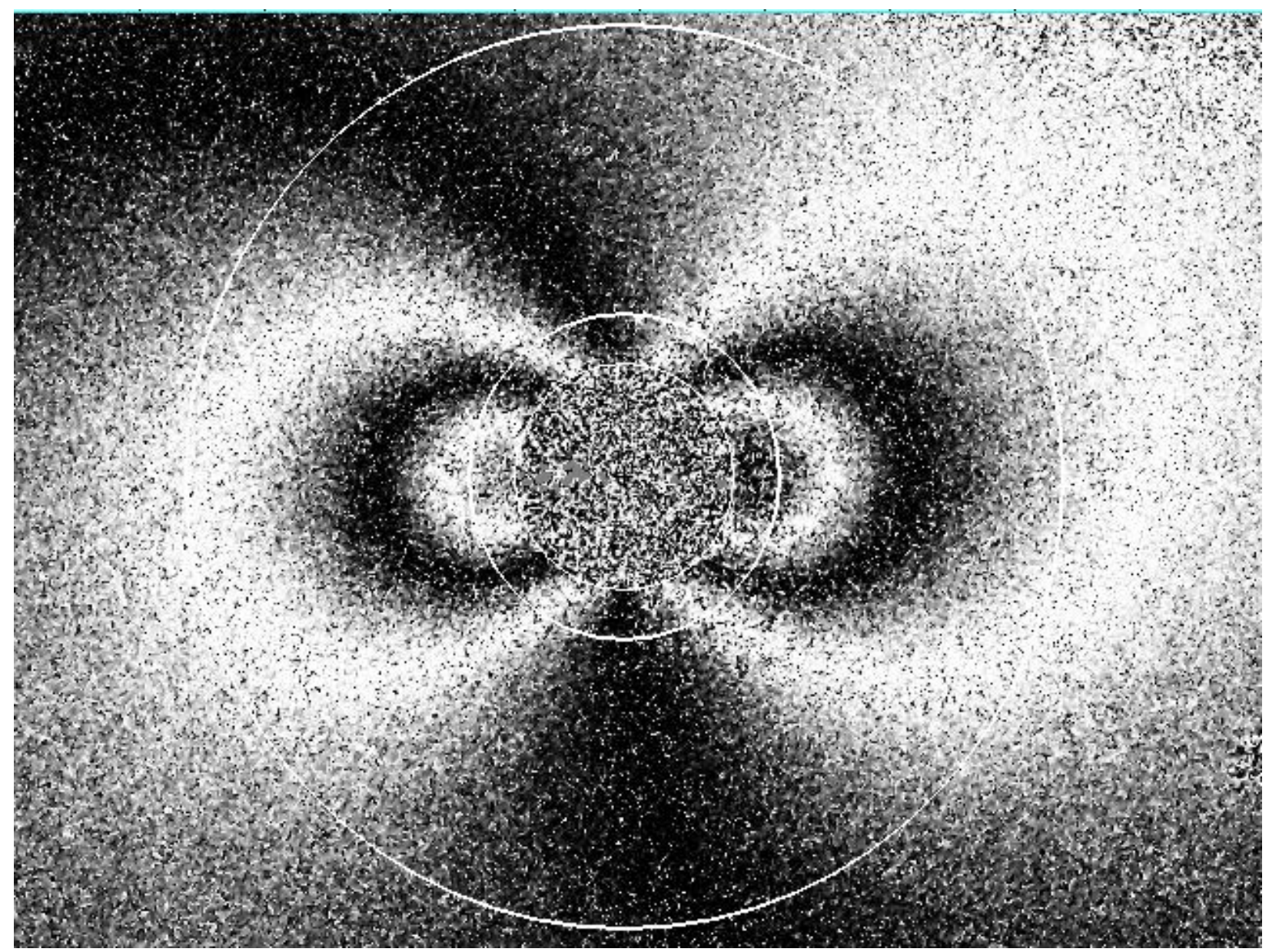

Figure 34: Fringe image derived from phase shift between reference set and measurement set.

The image above has clearly visible fringes, but there exists a certain level of noise in the image which appears as the black and white speckling. This comes from several sources, pixels that are over exposed or minute dust particles in the air or on the optical equipment. This can be minimized to a certain degree. If the pixel is bad it can be ignored and its value interpolated from its neighbours. This modified image is shown in figure 35 . 


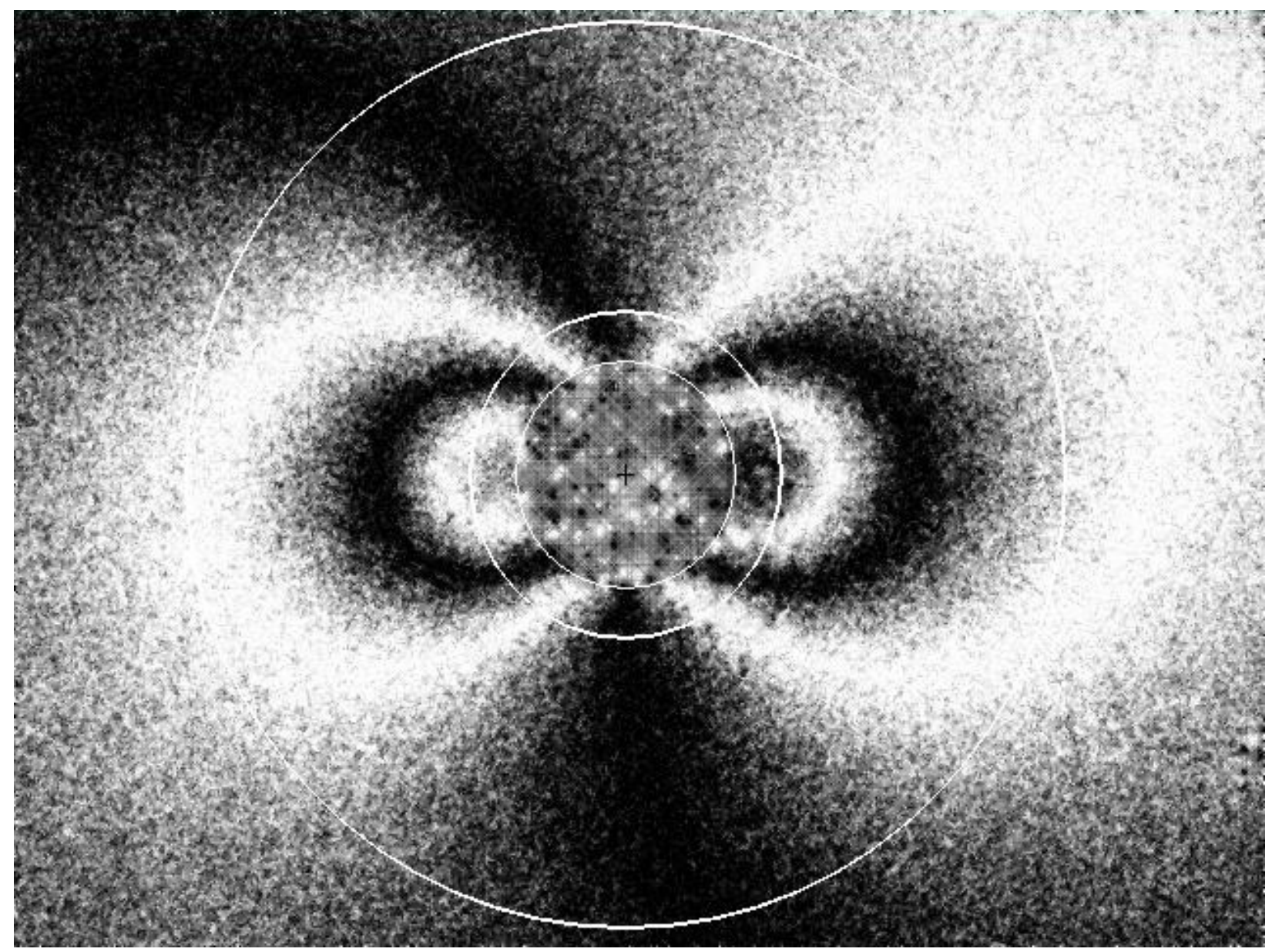

Figure 35: Fringe image with interpolation of bad pixels and outliers.

The phase images are converted into displacement maps and compared to the analytical solution. From this comparison the residual stresses relieved by drilling the hole can be calculated.

\subsection{Experimental Results}

Experimental tests were conducted to determine the validity of the newly derived equations. The equations were used with the experimental setup discussed above for ESPI measurement tests. If the stresses measured by the experimental method match the known stresses imparted by the specimen holder the method can be considered validated.

The test specimen is prepared as discussed in section 3.2. For this test the specimen is 6061-T6 aluminum with a modulus of elasticity of 71GPa. The thickness of the workpiece is $2.2 \mathrm{~mm}$ and the drill bit used had a radius of $1.2 \mathrm{~mm}$. 
Initially the stresses were calculated using the displacement equations directly. This produced substantial errors in the measured stress levels compared to the expected values. The cause of the errors was found to be the discrepancies very near the hole. The equations for case B produce the best results mainly over the radii used for strain gauge measurements. When using these equations very near the hole edge the magnitude of the stress response was incorrect. To avoid the errors caused by this the stresses were calculated as described in chapter 3.3 using the ASTM method. This produces results that assume the stress response of the thick case. The ratio of the response from the thick case to the specific intermediate case is then calculated. The results are then scaled appropriately by dividing the thick case by the ratio between the two. This produces results for the intermediate case. The results of these tests are shown below in figures 36 .

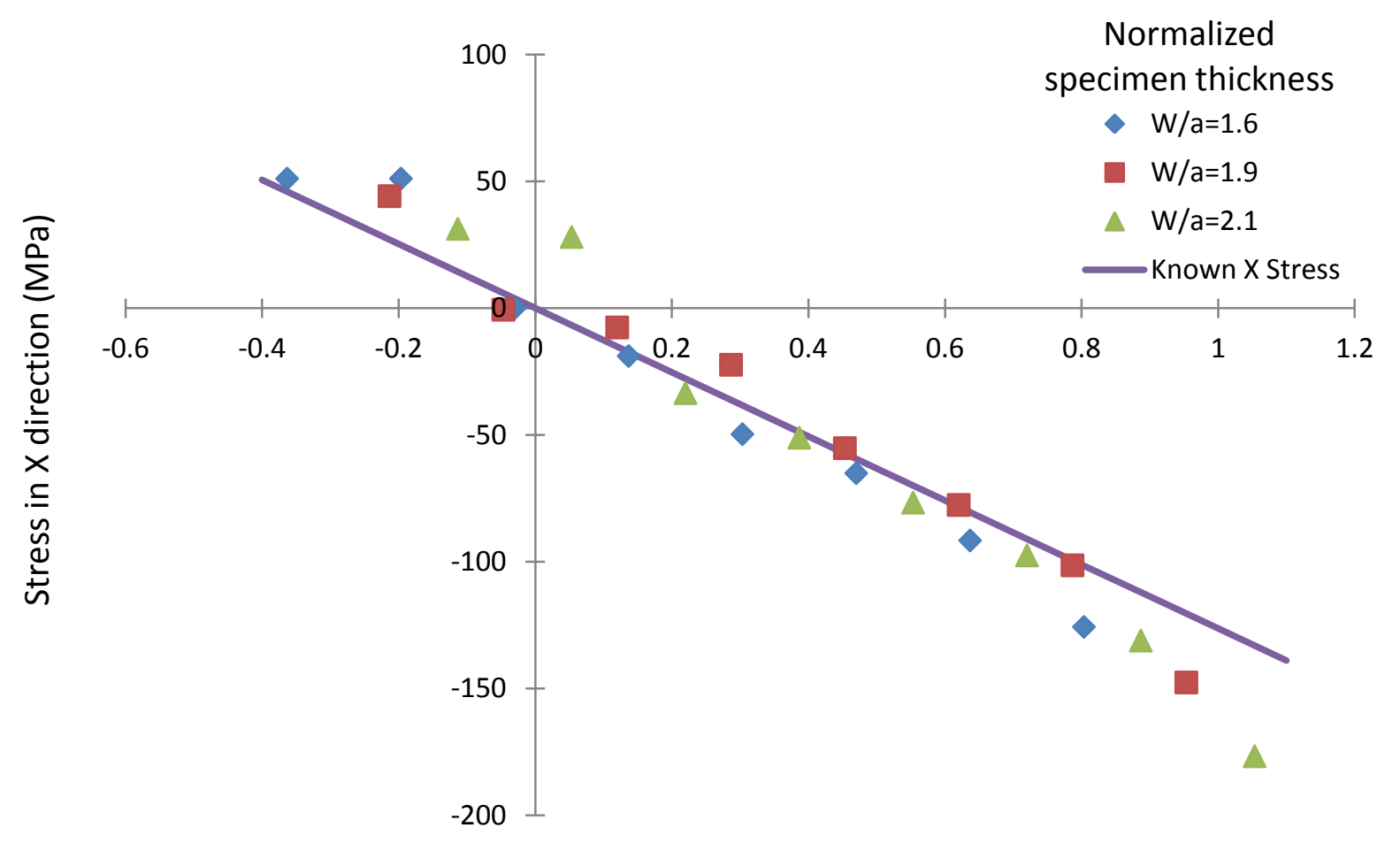

Distance from neutral plane (Y/a)

Figure 36: Experimental results for stress in the X direction compared to known value.

The tests chosen for this method are stress profiling measurements. These results allow us not only to compare the magnitude of the measurements, but also the trend. The results from several tests are compared the expected values shown on the graph as the solid line (figure 36). The stresses in the $\mathrm{X}$ direction compare quite well to the expected values. The first hole step contains slightly more error than subsequent steps. This is typical of the measurement and could be from several factors. Not detecting the surface 
exactly or surface damage from chips that scratch the surface could cause this error. Once the hole has been started, the chips will exit more away from the part and have a lesser chance of scratching the surface. Subsequent depth steps have slightly better accuracy because of this. As the hole depth increases, the stresses are being calculated deep in the part relative to the surface where the displacements are measured. This causes significant errors after the hole depth surpasses 1.4 times the hole radius. 


\section{Chapter 4 Conclusions}

The hole drilling method has historically been applied to cases where the workpiece was either very thin or very thick. This research addresses the intermediate thickness case and provides six major contributions.

1. This research provides insight into the behavior of the intermediate case and helps to develop a fundamental understanding of what is happening. Using finite element analysis the behavior of the specimen is examined visually over a wide range of hole depths and part thicknesses. Plots of the deformed shape and stress contour maps convey intuitively how the part reacts to the releasing of stresses as the hole is drilled. The bending phenomenon seen in the intermediate case had not been previously noted. These plots also illustrate how the deformations occur more locally as the part is thickened and the stiffness increases. The fact that the stress concentration of the B case is double that of the A case is clearly visible and helps to develop the modifications needed to account for this.

2. The intuitive understanding of the intermediate case provides the insight for the ring-disk approximation. This approximation helps to capture the complex behavior of the intermediate case in a way that can be easily understood. The ring-disk approximation also suggests that the response will not vary linearly between the thin and the thick case. The ring-disk approximation is central to the development of the mathematical model. This simplifying assumption works well for cases where the thickness of the part is modestly greater than the hole depth, but as the part thickness increases the assumption begins to break down.

3. The bending that occurs in the intermediate thickness provides an additional displacement contribution. This additional contribution makes the response not vary linearly between the thin and the thick cases. For cases where the thickness of the part is just greater than the hole depth, the response is actually greater than the response for the thin case. As the thickness increases in relation to the hole depth the response peaks and then starts to decrease as the thickness increases the stiffness and the bending diminishes. This nonlinear variation between the thin and thick cases necessitates more than a simple interpolation between the two cases.

4. A mathematical model is developed to compactly convey the surface displacements around the hole to the stress relieved by the hole drilling. The mathematical model comes from the insights that were gained from the finite element analysis and relies on the ring disk approximation. By finding the stiffness of the inner disk and outer ring the load distribution is found. The response is then calculated for the horizontal displacements and the bending contribution of the outer disk. These two components constitute the total displacement. For the B case this mathematical model is adjusted to account for the larger stress concentration factor. An interpolation is added as 
well to make this applicable to any thickness. The interpolation covers the transition between where the ring-disk approximation breaks down and the thick case. These mathematical equations can be used in ESPI measurements or can be converted to strain gauge calibration coefficients. This effectively extends the application of hole drilling residual stress measurement to workpieces of any thickness.

5. Stress profiling on the thin case was not previously possible. This severely limited the application of the method. Stress profiling can be applied to the thin case by treating the thin case as a series of intermediate cases and calculating the stress at each depth step. This method produces valid results, but the errors may be slightly more than stress profiling for the thick case because of the increased flexibility of the part.

6. The design and creation of an experimental setup is also a very valuable result of the project. The workpiece holder with the back removed creates an accurate way to test the intermediate case. The ESPI setup created for this experiment will be valuable to future research. The setup was created to be durable and easy to use and adjust while filling a minimum footprint so as to leave as much space to work with around it. The setup was designed with a focus on the stiffness of each part, a design consideration critical to achieving high quality experimental measurements. The circuit incorporates status LED's to help future troubleshooting and fuse protection to ensure long component life. A lock out key is also included to comply with laser safety guidelines.

Opportunities exist for future work on this research topic. This method assumes that the boundary between the inner disk and outer ring remains straight. A revision to the mathematical model that incorporated the nonlinearity of this line could improve the method. A model that included considerations for this would not need the modifications to the math for the B case. This could also help to increase the accuracy of the method and broaden its application. The cause of the nonlinear boundary at the hole edge is the stress concentration and local deformations. Developing a mathematical model that can account for the local deformations around the hole may allow the solution to be applied without the need for an interpolation to the thick case. 


\section{References}

[1] Withers P. J., Bhadeshia H. K. (2001). Overview Residual Stress, Part 1, Materials Science and Technology, Vol. 17, p. 355-365

[2] Spotts M. F., Shoup T. E., Hornberger L. E. (2003). Design of Machine Elements, eighth edition, New Jersey, Prentice Hall, p. 437-442

[3] Anderoglu, O. (2004). Residual Stress Measurement Using X-RAayDiffraction (masters thesis), Texas A\&M University

[4] Kudryavtsev, Y., \& Kleiman, J. (2004). Ultrasonic Technique and Device for Residual Stress Measurement, Integrity Testing Laboratory Inc., Ontario, Canada

[5] ASTM (2009). Standard Test Method for Determining Residual Stresses by the HoleDrilling Strain-Gage Method. E 837 - 08, Pennsylvania, American Society for Testing and Materials.

[6] Schajer, G. S. (2009). Hole-Drilling Residual Stress Measurements at 75: Origins, Advances, Opportunities, Experimental Mechanics, Volume 50, p. 245-253

[7] Schajer, G. S. (2001). Residual Stresses: Measurement by Destructive Testing, Encyclopedia of Materials: Science and Technology, Elsevier, pp. 8152-8158

[8] Yang, L., \& Ettemeyer, A. (2003). Strain Measurement by Three-Dimensional Electronic Speckle Pattern Interferometry: Potentials, Limitations, and Applications. Optical Engineering, Vol. 42, p. 5

[9] Schajer, G. S. (1981). Application of Finite Element Calculations to Residual Stress Measurements. Journal of Engineering Materials and Technology, Volume 103, p. 157163.

[10] Young, W. C., \& Budynas, R. G. (2002). Roark's Formulas for Stress and Strain. Two Penn Plaza, New York: McGraw-Hill Books, p. 623-629

[11] Schajer, G. S. (1993). Use of displacement data to calculate strain gauge response in nonuniform strain fields. Strain, Volume 29, p. 9-13.

[12] Vinson, J. A. (2005). Plate and Panel Structures of Isotropic Composites and Piezoelectric Materials Including Sandwich Construction, 1 edition, Norwell, MA, Springer, p. 67-93 
[13] Schajer, G. S. (1988). Measurement of Non-Uniform Residual Stresses Using the HoleDrilling Method. Part 1-Stress Calculation Procedures, Journal of Engineering Materials and Technology, Vol. 110, p. 338-343

[14] Schajer, G. S. (1988). Measurement of Non-Uniform Residual Stresses Using the HoleDrilling Method. Part 2-Paractical Application of the Integral Method, Journal of Engineering Materials and Technology, Vol. 110, p. 344-349

[15] Hariharan, P. (1992). Basics of Interferometry, Sydney, Australia: Avademic Press, Inc.

[16] An, Y. (2008). Residual Stress Measurement using Cross-Slitting and ESPI (masters thesis), The University of British Columbia

[17] Andersen, L. F. (2002). Experimental Method for Residual Stress Evaluation Through the Thickness of a Plate. Journal of Engineering Materials and Technology, Vol. 124, p. 428-433.

[18] Gong, H., Wu, Y., \& Liao, K. (2009). Research on Residual Stress Measurement Technology of Aluminum Alloy Plate Based on Modified Layer Removal Method. IEEE Spectrum, p. 3839-3842.

[19] Goodier, J. N. (1936). The Influence of Circular and Elliptical Holes on the Transverse Flexure of Elastic Plates. Philosophical Magazine, p. 69-80.

[20] Lobkova, N. A. (1967). The Equations from the Theory of Thin Nonuniform Plates in Cylindrical Coordinates. Prikladnaya Mekhanika, p. 22-26.

[21] Luh, G. C., \& Hwang, R. M. (1999). Measuring Non-Uniform Residual Stress in Thin Plates by a Proposed Hole-Drilling Strain Gauge Method. International Journal of Advanced Manufacturing Technology, p. 103-113.

[22] Wilson, J. W., Tian, G. Y., \& Barrans, S. (2006). Residual magnetic field sensing for stress measurement. Sensors and Actuators , p. 381-387. 